$1-1-1904$

\title{
Commercial fertilizers : complete report for 1904
}

\author{
J. H. Stewart
}

B. H. Hite

Follow this and additional works at: https://researchrepository.wvu.edu/ wv_agricultural_and_forestry_experiment_station_bulletins

\section{Digital Commons Citation}

Stewart, J. H. and Hite, B. H., "Commercial fertilizers : complete report for 1904" (1904). West Virginia Agricultural and Forestry Experiment Station Bulletins. 95.

https://researchrepository.wvu.edu/wv_agricultural_and_forestry_experiment_station_bulletins/95 
Digitized by the Internet Archive in 2010 with funding from

Lyrasis Members and Sloan Foundation 


\section{WEST VIRGINIA UNIVERSITY \\ AGRICULTURAL EXPERIMENT STATION \\ MORGANTOWN, W. VA.}

\section{Commercial Fertilizers}

\section{COMPLETE REPORT \\ FOR \\ I 904}

By J. H. STEWART AND B. H. HITE

[The Bulletins and Reports of this Station will be mailed free to any citizen of 'West Virginia upon written application. Address Director of Agricultural Experiment Station, Morgantown, W. Va.] 


\section{THE REGENTS OF THE WEST VIRGINIA UNIVERSITY}

Name of Regent. P. O. Address.

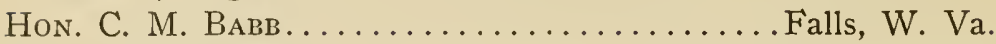

Hon. J. B. FInLEY ................. Parkersburg, W. Va. Hon. D. C. Gallaher..................... Charleston, W. Va.

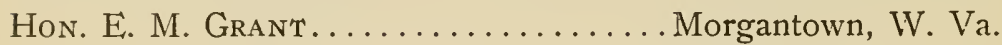
Hon. C. E. Haworth . . . . . . . . . . . Huntington, W. Va. Hon. C. P. McNell. ................Wheeling, W. Va. Hon. L. J. Williams................. Lewisburg, W. Va. Hon. T. P. JAcoss............... New Martinsville, W. Va. Hon. J. R. 'Trotter.................. Buckhannon, W. Va.

President of the Board of Regents........... J. R. TRoTTER President of the University.............. B. Purinton Treasurer..................... R. WHITEHII,

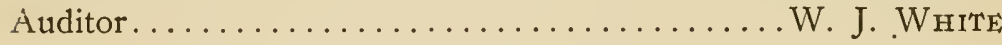

\section{STATION STAFF}

JAMES H. STEWART, A. M......... Director and Agriculturist BeRT H. Hite, M. S............ Vice Director and Chemist John L. SHELdon, PH. D.............. Plant Pathologist W. E. RumseY, B. S. Agr............. Entomologist in Charge Horace Atwood, M. S. Agr........... Assistant Agriculturist Chas. D. Howard, B. S............... Associate Chemist T. C. Johnson, M. S...... Botanist and Assistant Horticulturist

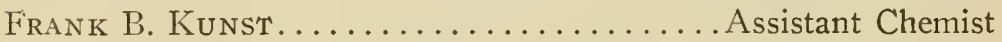
Frank F. Grout, B. S................. Assistant Chemist

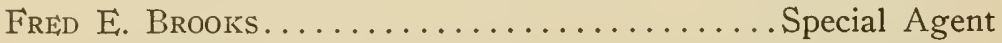

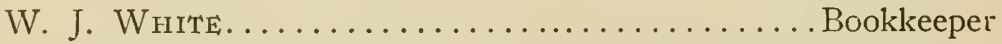

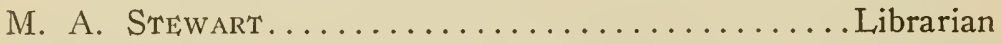

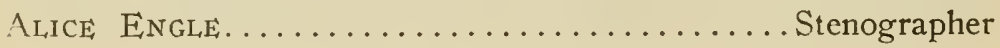




\section{COMMERCIAL FERTILIZERS}

The following pages contain a complete report of the work required by the fertilizer law of this state for the year I904.

Attention of purchasers of commercial fertilizers is directed to the Remarks to be found at the close of altogether too many of the paragraphs, in which the claims and findings for the various brands are reported. Farmers should make a note of the brands that fall short and refuse to buy them, unless they can be had at prices correspondingly low. Even then there is certainly nothing to be lost by purchasing from companies having more respect for their claims. For the farmer who is putting so much of the profits of his last crop into his prospects for the next, it is a matter of much concern that he be able to depend upon his fertilizers with a far greater degree of certainty than the shortages of some concerns can possibly inspire.

Of course, it is always claimed that "everybody makes mistakes sometimes," so a manufacturer may make a mistake in add. ing too little of some particular constituent, and this is all true. It is also true that the mistakes in question will be more likely to occur in proportion to the eagerness of the manufacturer to cut as close as possible to the minimum figure. This office cannot be accused of captiousness in regard to an occasional shortage where the quantity of other constituents bear evidence of good faith. The shortages to be noted in this report are more largely a matter of quality than of the quantity of the materials used. They are, therefore, the more difficult to construe as accidental. Manufacturers do not mistake kainit or low-grade muriate for high-grade sulphate; or garbage tankage for dried blood or nitrate of soda. Yet there are a number of firms who, for example, claimed highgrade sulphate in a number of brands, and who (so far as can be learned from such samples as could be found by the inspectors), 
used it in none. Indeed, there are only two or three concerns whose unequivocal claims for high-grade sulphate of potash coulrl be verified in all cases, while one large concern and two or three smaller ones are using, in-some cases, materials the chlorine equivalent of which far exceeds that of the lowest grade source of potash claimed.

It should be observed that the claims of manufacturers, given in this report, are the clearest that could be obtained, the affidavits in some cases having been returned several times for "more explicit statements." This, of course, does not apply to many firms whose statements are invariably straightforward and to the point. It should be sufficient to simply submit the following pages of this report with their verbatim reproductions of the statements made in the affidavits and leave it to the purchasers and users of commercial fertilizers to discover, if they can, some reason for the certain equivocal statements, other than a well defined determination to avoid the disclosure of anything that might aid a farmer in selecting a fertilizer, and to see to it that this little is so effectually garbled as to stand a chance of misleading him. Take, for example, the claim that the potash is derived from "sulphate muriate and kainit." It is, of course, a safe guess that kainit was the material to be used, but why mention the other higherpriced materials? It is the opinion of the writers that the higherpriced materials were named in the affidavits for the purpose of leading the purchaser to believe they would be found in the fertilizers. It is the privilege of the reader to find some other motive, if he can. Some of the same firms drafting misleading clains herein reported have been loud in their disapproval of the provision of the statute requiring a disclosure of the materials used, on the ground that the farmers neither know nor care anything about such matters. But why, then, be so careful to deceive them? It ought to be as easy to state the facts in one word as a falsehood in four. The truth of the matter is that the farmers do know and do care, and will very quickly and very practically turn to account any information they can get in regard to such matters.

The arlvice of this office to purchasers of commercial fertilizers. 
in this state is: First, as to the quantity of any constituent claimed, be guided by the minimum guarantee; second, as to the quality of any constituent claimed, be guided by the lowest grade material mentioned, unless the part, derived from each material mentioned, is distinctly stated. For example, the claim above referred to, that the potash is derived from sulphate muriate and kainit, will probably mean just what the inspector found, while the claim "one-fourth from muriate and three-fourths from kainit" will probably mean just what it says. It is safe to assume that low-grade materials will not be mentioned, unless they are going to be used, and there is no reason for refusing to state the proportion in which they will be used except that the claim would look better without such a statement.

For the year 1905 all manufacturers will be asked to state what part of the various constituents claimed is derived from the materials named. It is perfectly safe to predict that many manufacturers will comply gladly, others grudgingly, while others will make all the trouble they can, but the farmers shall know who the manufacturers are that are willing to let their fertilizers stand on their own merits, also those who consider it advisable to let the purchaser have as little information as possible.

The statements in regard to the materials used are certainly no more difficult to make than those which manufacturers usually insist upon making in big type all over the bags. It requires a chemical analysis to determine the amount of the constituents claimed, while the man behind the wheel-barrow in the mixingroom would probably know the materials used.

The information which our law demands in regard to the materials used is absolutely necessary not only that fertilizers may be purchased at a fair price but also that they may be used to best advantage. The requirements in question are objectionable to some concerns because they strike at the root of an otherwise splendid opportunity to sell inferior materials at the price of the best. In this connection it will be interesting to read a paragraph from the Virginia Report on Commercial Fertilizers, which is quoted in this report under the head of "Valuations." The Virginia control is urging farmers not to be mislead by high sound- 
ing names on the bags, but to be guided strictly by the amount of the constituents" claimed. This sounds like an echo from the dim distant past, and to thousands of farmers in this state it may recall, very vividly, the good old days when the old law in this state, like the present Virginia law, provided an unscrupulous manufacturer (under official sanction) with finer opportunities for cheatery than have ever been concocted by anybody connected with the fertilizer business. Not a word in regard to the quality of the materials used, although this may easily be a matter of much greater concern than the quantity. What an opportunity for ground leather and hair, with "ammonia $161 / 2$ cents per pound!" To the Virginia law almost all the materials look alike, if only they can contribute to the amount of some constituent claimed. A thousand and one dealers and farmers in this state should readily understand from the above why it is they were interviewed by agents of certain fertilizer concerns in regard to the presumably nonsensical and otherwise objectionable features of the present West Virginia law. They will also understand why it was they were expected to listen to a disclosure of the innumerable "advantages" of the present Virginia law. The thing that was worrying these representatives, and the only thing, was that the "advantages," that is to say, the real advantages to them, so liberally provided by the Virginia law, don't go in IVest Virginia, unless the parties attempting them swear falsely. Of course, the representatives in question are always careful to steer clear of such "advantages" as those just mentioned, almost any other excuse for fault-finding being urged instead, but one excuse is as good as another when everybody knows the real reason.

West Virginia has the best fertilizer law in the United States, excepting, perhaps, California, where practically the same law was recently enacted. It is the law which makes deception in the fertilizer business the most difficult. It is for this reason, and for this reason alone, the most distasteful to a number of fertilizer concerns with whom it would seem a fertilizer law is simply a proposition, in the nature of the case, antagonistic, and that the problem is to beat it. It is the hardest law to beat. It would seem that certain manufacturers discovered this fact shortly after 
this law was enacted and realized very clearly how far the usual methods must fall short, for, whether at their wits' end or not. they resorted to tactics which, in the experience of those connected with this office, were certainly unique. Representatives of the concerns in question, having been much announced and having duly arrived, did most emphatically lay down the proposition to boycott the state, if those in charge of the fertilizer control attempted to carry out the requirements in regard to the materials used. These, like all other requirements of the law, have been carried out. The concerns in question have not boycotted the state, although it is understood they did try to do so. All this, to avoid complying with a law so fair, so generous, that anybody may sell as a fertilizer anything on earth he pleases, provided only that he sells what he claims to sell. The law admits of no other construction. No other construction has ever been attempted by this office. (Mention this fact when interviewed again.)

It is to be regretted that such comments as the above have to be made. Those in charge of this office have always wished the opportunities for deception peculiar to the fertilizer business might find no occasion for comment in these reports. Few sets of men can claim a keener appreciation for the part that must be played by commercial fertilizers in the building up of soils, so long and so wantonly mistreated as those in which the hillsides of this state abound. Few sets of men have had better reason for a higher regard for the business integrity and business pride of the majority of fertilizer manufacturers, for some of those connected with this office were witnesses to the operations of the old law of this state, which to all intents and purposes was the same as the present Virginia law, and which did not stop with permitting or even encouraging the use of inferior materials, but which must have taxed to the limit the business integrity of any firm doing business with it to avoid using low-grade materials in self-defense. But when, of all the brands of a few concerns that can be found on sale in the state, 30 per cent. to 50 per cent. fall short in quantity or quality of the constituents claimed, we submit the matter demands more than a passing mention. 
Valuations are not required by the fertilizer law of this state, because they can never be fair for the period covered by a report, and in justice should not be enforced by law. It should be sufficient that the law provide the purchaser of commercial fertilizers with such information as may be necessary to enable him to avoid the possibilities for deception peculiar to the fertilizer business. Having assured him as to what he is buying, there is no more reason for fixing by law a price for fertilizers than for rocking chairs, hobby horses or anything else that might be mentioned. The purchaser must keep himself informed as to prices. That is the only way. However, the valuations, adopted by adjoining states during 1904, may be of interest.

\section{VIRGINIA.}

"To have a basis for comparing the values of different fertilizers it is necessary to assign values to the three valuable constituents of fertilizers, i. e., phosphoric acid, ammonia, and potash. The raw materials used by manufacturers in preparing their fertilizers are bought on a basis of the per cent. of the valuable constituents contained in the different materials. It is evident, then, that the value of a fertilizer is measured by the current price of the raw materials contained in it, plus the cost of manipulation. The relative values as put down in the tables have nothing to do with the crop-producing value, but are estimates of the commercial value of the valuable fertilizing ingredients contained in the fertilizer. These values are only approximate, as the cost of fertilizer'materials are liable to change like other commercial products, and it would be impossible to assigu values which would represent the cost during an entire season. They are based on a careful examination of trade conditions and are believed to fairly represent the cost during an entire season. They are based on a careful examination of trade conditions and are believed to fairly represent the cost of putting the fertilizer on the market. They are most valuable as a means of comparison, and should be mainly used for that purpose.

"The farmer should study the analyses of the fertilizers and make his purchases accordingly, and not be led off by any high-sounding name. He should buy strictly by the amount of fertilizing ingredients contained in a fertilizer, and not by the name, as the ingredients are what make the crops grow, and not the name." 


\section{Valuations for 1904 .}

"The figures which will be used for calculating values in this Bulletin are slightly different from those used last year, and are as follows:

\section{In Unmixed or Raw Materials.}

Cts. per $1 b$.

Available Phosphoric Acid in Acid Phosphate.......... .04

Phosphoric Acid in Animal Bone................ $03 \frac{1}{1 / 2}$

Phosphoric Acid in Basic Slag Phosphate.......... .041/2

Phosphoric Acid in Orchilla Guano.............. . . . . . . . .

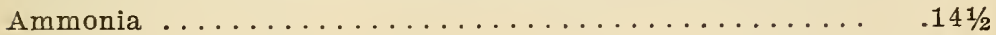

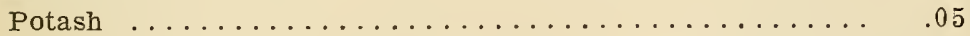

In Mixed Fertilizers.

Cts. per $\mathrm{lb}$.

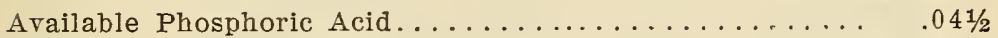

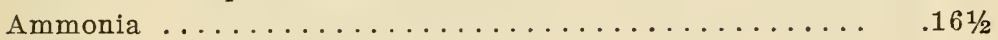

Potash ............................. $051 / 2$

\section{How Relative Values Are Calculated.}

"In the calculation of relative values it is only necessary to remember that so many per cent. means the same number of pounds per hundred, and that there are twenty hundred pounds in one ton ( $100 \times 20$ equals 2,000 pounds, equals one ton). Therefore, to find the value per ton, multiply the per cent. of each ingredient by 20 , which gives the number of pounds of each ingredient in a ton, then multiply this result by the price per pound of that ingredient, then add up the figures thus obtained for each ingredient, and the sum is the relative value per ton.

As an example, take an 8-2-2 fertilizer, which means that the fertilizer contains 8 per cent. available phosphoric acid, 2 per cent. ammonia and 2 per cent. potash.

The calculation is as follows:

Per cent $=$ lbs. per 100

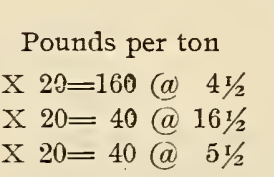

Value per

8 Phosphoric acid. ton

2 Ammonia

2

Potash.

6.60

2.20

Total

$\$ 16.00$ 


\section{PENNSYLVANIA.}

"The schedule for 1904 as a whole is as follows:

\section{Schedule of Values for Fertilizer Ingredients, 1904.}

Nitrogen:

Cts. per $1 \mathrm{~b}$.

In ammonia salts.....................171/2

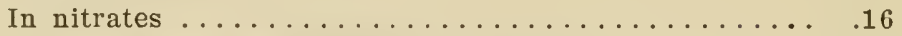

In meat, dried blood and mixed fertilizers. . . . . . . . . 171/2

In cotton seed meal and castor pomace............ . . . . . . . . . .

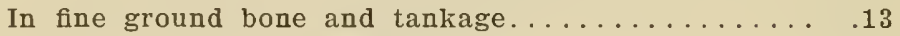

In coarse bone and tankage.................11

\section{Phosphoric Acid:}

Soluble in water, in bone fertilizers. . . . . . . . . . 04 1/2

Soluble in water, in rock fertilizers............... 03

Soluble in ammonium citrate, in bone fertilizers..... . .04 1/2

Soluble in ammonium citrate, in rock fertilizers. . . . . . .03

Insoluble in ammonium citrate, in bone fertilizers... . .02

Insoluble in ammonium citrate, in rock fertilizers . . . . . .011/2

In fine bone, tankage and fish............. .03

In coarse bone and tankage................. . . $2 \frac{11 / 2}{2}$

In sotton seed meal, castor pomace and wood ashes.... .04

\section{Potash:}

In high-grade sulphate or in forms free from muriate. . .05

As muriate ................... 04 1/4

The trade-values in the following schedule have been agreed upon by the Experiment Stations of Massachusetts, Rhode Island. Connecticut, New York, New Jersey and Vermont, as a result of study of the prices actually prevailing in the large markets of these states.

These trade-values represent, as nearly as can be estimated, the average prices at which, during the six months preceding March, the respective ingredients, in the form of unmixed raw materials, could be bought at retail for cash in our large markets. These prices also correspond (except in case of available phosphoric acid) to the average wholesale prices for the six months preceding March, plus about 20 per cent., in case of goods for which there are wholesale quotations.

\section{Trade-Values of Plant-Food Elements in Raw Materials and} Chemicals.

1903.

Cts. per $1 \mathrm{~b}$.

Nitrogen in ammonia salts................... 171/2

Nitrogen in nitrates..................... 15 
Organic nitrogen in dry and fine-ground fish, meat and

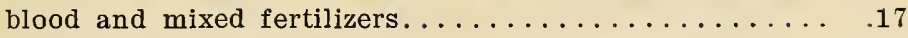

Organic nitrogen in fine-ground bone and tankage....... .161/2

Organic nitrogen in medium bone and tankage......... .12

Phosphoric acid, water-soluble.................. $04 \frac{11 / 2}{2}$

Phosphoric acid, citrate-soluble (reverted) .......... .04

Phosphoric acid, in fine-ground fish, bone and tankage..... .04

Phosphoric acid, in cotton seed meal, castor-pomace and

ashes .......................... 04

Phosphoric acid, in coarse fish, bone and tankage....... .03

Phosphoric acid, in mixed fertilizers, insoluble in ammonium

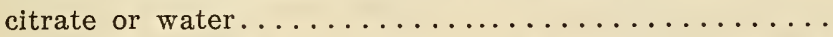

Potash as high-grade sulphate, in forms free from muriates

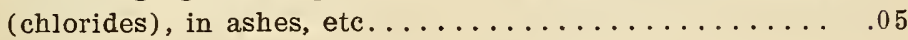

Potash, in muriate..................... $04 \frac{11 / 2}{2}$

Cost of One Pound of Plant-Food in Fertilizers as Purchased by Consumers.

In the table below'we present figures showing the average cost to the purchaser of one pound of plant-food in different forms in mixed fertilizers.

Average Cost of One Pound of Plant-Food to Consumers in Mixed Fertilizers.

Nitrogen ......................23.00 cents

Phosphoric acid (available) ............. 5.75 cents

Potash ........................ 6.10 cents

The above quotations will serve to show the prices at which it was believed fertilizers ought to be bought in the several states mentioned. They will also serve to show the wisdom of our own lawmakers in declining to enforce such opportunities for embarrassment and unfairness as the usual "valuation" so liberally provides. Farmers must keep themselves informed as to such matters. It's the only way, and it will be easy enough whenever they demand that the farm papers coming regularly to their doors shall provide quotations in regard to fertilizers as promptly, regularly and accurately as the press of other industries provides quotations on commodities of like importance.

In connection with the Remarks which have to be made in regard to certain brands, the foot notes in regard to the availibility of nitrogen and the following table giving the important sources of potash, will be of service: 


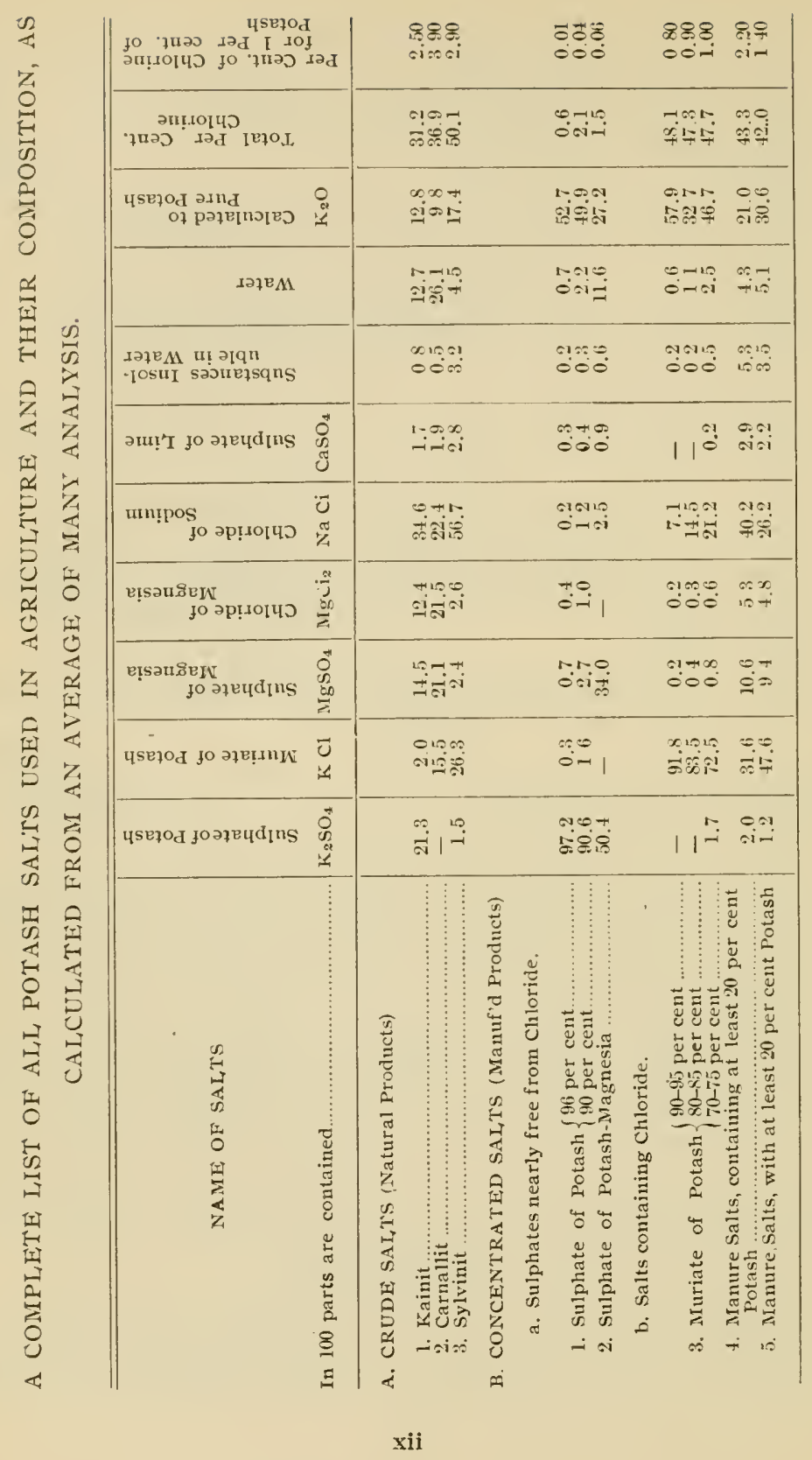


The quality of the materials used in the fertilizers supplied to this state during the past year is, in the great majority of cases, very satisfactory, as shown by the following data concerning nitrogen availability and chlorine:

In Bones:

NITROGEN AVATLABILITY.

Highest nitrogen availability test.........994 per cent

Lowest " " " "

Average " " " " "

In Mixed Fertilizers:

Highest nitrogen availability test..........95 per cent

Lowest " " " " $" \ldots \ldots \ldots \ldots \ldots \ldots 66$ per cent

Average " " " " $" \ldots \ldots \ldots \ldots \ldots .85$ per cent

Range of majority, 80 per cent. to 90 per cent.

\section{CHLORINE.}

Per cent. of chlorine for 1 per cent. potash in fertilizers for which kainit was the guaranteed source, or where it was evident that this material had been used:

Highest per cent. Chlorine for 1 per cent. potash... 10.7 per cent. Lowest per cent. Chlorine for 1 per cent. potash..... 1.6 per cent Average per cent. Chlorine for 1 per cent. potash.... 3.69 per cent Average actual per cent. Chlorine for 1 per cent.

potash (in kainit) ................ 2.5. 0 per cent

The above data show that the materials used as sources of nitrogen were, on the whole, better than those used as sources of potash, and that in both cases the averages would have made ? much better showing but for the fertilizers provided by a few concerns. It was with these few concerns in mind that some of the preceding paragraphs were written, and they were written for the purpose of whipping the said concerns into line or driving them out of the state. It would be, by no means, the first instance of the sort, nor the first time the farmers of the state have been saved many times the cost of a fertilizer control. Recall, for example, the Natural Plant Food affair, which is suggested by the endeavors now in progress to get a similar stuff into the state without complying with the law. The material in question is "floats," or untreated but ground phosphate rock. Following closely on the heels of the advertising it has recently received in certain quarters, this material is again ready for market. The excuse assigned for not complying with the law is that " $i t$ is not a fertilizer." This claim breaks all records to date. 
The following pages devoted to the claims and findings for the various brands should, on the whole, be a source of satisfaction to the purchasers of commercial fertilizers in this state. If we could but avoid, exclude or otherwise get rid of the brands of a few concerns that should be already well known from former reports and that may be counted on the fingers of one hand, the cases in which straightforward claims as to quantity and quality could not be verified are almost excusably small. The law is accomplishing the thing for which it was intended. Farmers in purchasing commercial fertilizers in this state run as little risk of being cheated as in the purchase of almost any other commodity. There is reason to believe that during the past year the same amount of money bought more and better fertility than ever before in the state. Of course, competition unmolested tends to tighten, but for a long time the old law provided an ugly barrier to competition in that the high registration fee distinctly favored the large manufacturers and those with a large business already well established in the state. Since the new law practically abolished the registration fee, competition has been accomplishing just what was to be expected. This office has gone to no little pains to ascertain the prices at which the same fertilizers are being sold in this and adjoining states. It may be stated with all the assurance that could reasonably obtain in such matters that manufacturers' prices to agents in West Virginia are certainly as good as to agents in any adjoining state.

Attention of agents is called to Section 8 of the fertilizer law, as follows: "And it shall be the duty of every agent and dealer in fertilizers or manures within the meaning of this act in this state, when ordering consignments thereof, to promptly notify the Director of the said Experiment Station of the amount ordered, and the place and approximate time of delivery of said fertilizers and manures." Excepting negligence, which is inexcusable, there can be but one reason for failing to comply with this provision of the law, and that is a willingness to avoid inspection. When finding fertilizers on sale that have not been reported the inspectors will place the matter at once in the lands of the Prosecuting Attorney of the county in which the offense was committed. 


\title{
RESULTS OF THE
}

\section{Inspection of Commercial Fertilizers}

\author{
FOR 1904
}

GOODS OF

\section{AMERICAN AGRICULTURAL, CHEMICAL COMPANY,}

New York ANd Baltimore.

4952. A. A. C: CO.'S REGULAR CORN FERTILIZER. J. W. Minear, Parsons. Guarantee: Phosphoric acid, soluble, 4 ; reverted, 2 ; insoluble, I ; total, 7 ; available, 6 ; potash, 3 ; phosphoric acid from dissolved phosphate rock; potash from kainit and agricultural salt. Found: Phosphoric acid, soluble, 4.03; reverted, 2.97; insoluble, 0.22 ; total, 7.22 ; available, 7 ; potash, 3.08; *chlorine, 5.I.

Remark: Chlorine excessive.

* This represents the proportion of chlorine corresponding to one per cent. of potash in the fertilizer. The actual percentage of chlorine may be found by multiplying the above by the actual percentage of potash present. Chlorine is a constituent of muriate of potash, of common salt and of certain compounds of lime and magnesia. When high-grade sulphate is used as the source of potash the fertilizer should contain no more than traces of chlorine. The quantity of the latter increases directly with the inferiority of the source employed and its estimation serves approximately as an indication of such source. Where kainit is guaranteed and the chlorine exceeds 4.5 , it may be assumed that an inferior grade of this material has been used.

(See page 12.) 
4974. A. A. C. CO.'S REGULAR CORN FERTILIZER. Bishop \& Barbe, Jane Lew. Guarantee: (As above.) Found: Phosphoric acid, soluble, 2.53; reverted, 4.66; insoluble, 0.23 ; total, 7.42 ; available, 7.19; potash, 2.84 ; chlorine, 8.o.

Remark: Chlorine excessive.

4982. A. A. C. CO.'S GENUINE GERMAN KAINIT. T. P. Licklider, Martinsburg. Guarantee: Potash, I2 ; potash from kainit. Found: Potash, II.82; chlorine, 3.2.

4968. GENUINE GERMAN KAINIT. Washington \& Alexander, Charles Town. Guarantee: (As above.) Found: Potash, I2.78; chlorine, 3.I.

5040. A. A. C. CO.'S GEM ALKALINE PHOSPHATE. T. B. Drummond, Buckhannon. Guarantee: Phosphoric acid, solu ble, 4 ; reverted, 2 ; insoluble, I ; total, 7 ; available, 6; potash, 3 ; phosphoric acid from dissolved phosphate rock; potash fronı kainit and agricultural salt. Found: Phosphoric acid, soluble, I.87 ; reverted, 4.86; insoluble, 0.36; total, 7.09 ; available, 6.73; potash, 2.93 ; chlorine, 6.6 .

Remark: Chlorine excessive.

5145. A. A. C. CO.'S GEM ALKALINE PHOSPHATE. R. Hunter, Berkeley Springs. Guarantee: (As above.) Found. Phosphoric acid, soluble, I.30; reverted, 5.61; insoluble, 0.65 ; total, 7.56; available, 6.9I ; potash, 3.09 ; chlorine, 5.2.

Rcmark: Chlorine excessive.

5043. A. A. C. CO.'S GEM ALKALINE PHOSPHATF. C. W. Siever, Keyser. Guarantee: (As above.) Found: Phosphoric acid, soluble, I.93; reverted, 4.86; insoluble. 0.37 ; total, 7.I6; available, 6.79; potash, 3.16; chlorine, 7.I.

Remark: Chlorine excessive.

5053. A. A. C. CO.'S BONE MEAL. C. W. Siever, Keyser. Guarantec: Phosphoric acid, insoluble, I4 ; nitrogen, I.65; 
phosphoric acid from bone; nitrogen from animal bone. Found: Phosphoric acid; total, I5.39; nitrogen, I.75; *availability of nitrogen, 85 .

5070. A. A. C. CO.'S I6 PER CENT. SUPER PHOSPHATE. Philippi Mill Co., Philippi. Guarantee: Phosphoric acid, soluble, I4; reverted, 2 ; insoluble, I ; total, I7 ; available, I6; phosphoric acid from dissolved phosphate rock. Found: Phosphoric acid, soluble, I3.28; reverted, 3.6I ; insoluble, 0.I9; total, I 7.08 ; available, I6.89.

5099. A. A. C. CO'S I6 PER CENT. SUPER PHOSPHATE. Philippi Mill Co., Philippi. Guarantee: (As above.) Found: Phosphoric acid, soluble, I4.Io; reverted, 4.23 ; insoluble, 0.4I ; total, I8.65; available, I8.24.

507I. A. A. C. CO.'S FINE GROUND BONE. Philipp1 Mill Co., Philippi. Guarantee: Phosphoric acid, insoluble, 23; nitrogen, 2.47; phosphoric acid from bone; nitrogen from animal bone. Found: Phosphoric acid, total, 23.8I ; nitrogen, 2.52; availability of nitrogen, 94 .

5127. A. A. C. CO.'S FINE GROUND BONE. George A. Herring, Kingwood. Guarantee: (As above.) Found: Phos-

*The figure for the availability of nitrogen represents that proportion of the total nitrogen of the fertilizer which is immediately available to the plant as food. The test employed is a modification of Street's neutral permaganate method. While the results obtained can be considered as but an approximation to the true values as indicated by actual field tests, nevertheless they are valuable as serving to show the relative availability of the nitrogen in various brands of fertilizers. An availability of 85 or above is considered high, $80-85$ medium, below 75 , low. The availability of nitrogen in bone meals is necessarily higher than in mixed goods unless nitrate is used in the latter. 
phoric acid, total, 24.47; nitrogen, 2.36; availability of nitrogen, 93.

Remark: Nitrogen low.

5074. WHEELER'S UNAMMONIATED WHEAT SPECIAL. C. A. Anglin, Philippi. Guarantee: Phosphoric acid, soluble, 9; reverted, 3 ; insoluble, I ; total, I3; available, I2: phosphoric acid from dissolved phosphate rock. Found: Phosphoric acid, soluble, I0.99; reverted, 4.78 ; insoluble, O.I2; total, I 5.89 ; available, 15.77.

5OII. BRADLEY'S BEAN AND POTATO PHOSPHATE. O. R. Carmen, Wellsburg. Guarantee: Phosphoric acid, soluble, 6; reverted, 2 ; insoluble, I ; total, 9; available, 8; potash, 4; nitrogen, 0.80 ; phosphoric acid from dissolved phosphate rock and dissolved animal bone; nitrogen from dissolved fish, tankage and other animal. matter; potash from kainit and muriate of potash. Found: Phosphoric acid, soluble, 5.13; reverted, $3.5^{8}$; insoluble, 2.05 ; total, 10.76; available, 8.01 ; nitrogen, 0.98 ; potash, 3.25 ; availability of nitrogen, 88 ; chlorine, 2.4 .

Remark: Potash low.

5058. CANTON GAME GUANO. Dean \& Reeves, Fairmont. Guarantee: Phosphoric acid, soluble, 6; reverted, 2 ; insoluble, I ; total, 9 ; available, 8 ; nitrogen, I.65; potash, 2 ; phosphoric acid from dissolved phosphate rock and dissolved aninal bone; nitrogen from fish, bone, tankage and other animal matters: potash from kainit. Found: Phosphoric acid, soluble, 4.23; reverted, 4.19; insoluble, 0.75 ; total, 9.I7; available, 8.42 ; nitrogen. 2.OI : potash, 2.5I; availability of nitrogen, $9 \mathrm{I}$; chlorine, 3.O.

4976. CANTON POTATO MANURE. Bishop \& Barbe, Jane Lew. Guarantee: Phosphoric acid, soluble, 4; reverted, 2 ; insoluble, I ; total, 7 ; available, 6 ; nitrogen, I.23; potash, 5 ; phosphoric acid from dissolved phosphate rock and dissolved animal bone; nitrogen from dissolved fish, tankage and other animal 
matter; potash from kainit and muriate of potash. Found: Phosphoric acid, soluble, 4.89; reverted, I.57; insoluble, 0.99; total, 7.45 ; available, 6.46 ; nitrogen, I.35; potash, 4.94 ; availability of nitrogen, 90 ; chlorine, 2.I.

5035. CANTON POTATO MANURE. Armstrong, Chrislip, Day \& Co., Buckhannon. Guarantee: (As above.) Found: Phosphoric acid, soluble, 3.I7; reverted, 3.44; insoluble, I.24; total, 7.85 ; available, 6.6I ; nitrogen, I.4I ; potash, 4.78 ; availability of nitrogen, 78 ; chlorine, I.4.

5059. CANTON POTATO MANURE. Dean \& Reeves, Fairmont. Guarantee: (As above.) Found: Phosphoric acid, soluble, 4.66; reverted, I.99; insoluble, I.or ; total, 7.66; available, 6.65 ; nitrogen, I.46; potash, 5.52; availability of nitrogen. 88 : chlorine, 2.0.

5066. CANTON POTATO MANURE. J. M. Hagerty, Farmington. Guarantee: (As above.) Found: Phosphoric acid, soluble, 4.48; reverted, 2.96; insoluble, I.23; total, 8.67; available, 7.44 ; nitrogen, 1.57 ; potash, 4.46 ; availability of nitrogen, 86; chlorine, I.3.

Remark: Potash low.

5052. CANTON POTATO MANURE. C. W. Siever, Keyser. Guarantee: (As above.) Found: Phosphoric acid, soluble, 3.89 ; reverted, 2.67 ; insoluble, 0.86 ; total, 7.42 ; available, 6.56 ; nitrogen, I.38; potash, 5.39; availability of nitrogen, 76 ; chlorine, 2.2.

4977. CANTON SOLUBLE BONE AND POTASH. T. P. Licklider, Martinsburg. Guarantee: Phosphoric acid, soluble, 8 ; reverted, 2 ; insoluble, I ; total, I I ; available, Io; potash. 2; phosphoric acid from dissolved phosphate rock; potash from kainit. Found: Phosphoric acid, soluble, 6.37; reverted, 5.06; insoluble, 0.70; total, I I.I3; available, I0.43; potash, 2.09 ; chlorine, 4.I. 
5033. CANTON SOLUBLE BONE AND POTASH. Armstrong, Chrislip, Day \& Co., Buckhannon. Guarantee: (As above.) Fonnd: Phosphoric acid, soluble, 7.15; reverted, 3.42; insoluble, 0.42 ; total, I0.99; available, I0.57 ; potash, 2.14 ; chlorine, 4.I.

5I59. CANTON SOLUBLE BONE AND POTASH. Washington \& Alexander, Charles Town. Guarantee: (As above.) Fonild: Phosphoric acid, soluble, 7.I6; reverted, 4.I8; insoluble, 0.72 ; total, I2.06; available, II.34; potash, I.86; chlorine, 3.2 .

5036. BAKER'S DISSOLVED S. C. BOVE. Armstrong, Chrislip, Day \& Co., Buckhannon. Guarantee: Phosphoric acid, soluble, I2 ; reverted, 2 ; insoluble, I ; total, I5; available, I4; phosphoric acid from dissolved phosphate rock. Found: Phosphoric acid, soluble, II.82; reverted, 4.69 ; insoluble, 0.50 ; total, I 7.0I ; available, I6.5I.

5063. BAKER'S DISSOLVED S. C. BONE. J. M. Hagerty, Farmington. Guarantee: (As above.) Found: Phosphoric acid, soluble, 8.52 ; reverted, 7.44 ; insoluble, 0.77 ; total, 16.73; available, I 5.96 .

49So. BAKER'S DISSOLVED S. C. BONE. T. P. Licklider, Martinsburg. Guarantee: (As above.) Found: Phosphoric acid, soluble, I0.93; reverted, 4.30; insoluble, 0.67 ; total, I 5.90 ; available, I 5.23 .

5162. BAKER'S DISSOLVED S. C. BONE. Washington \& Alexander, Cliarles Town. Guarantee: (As above.) Found: Phosphoric acid, soluble, I0.34; reverted, 4.19; insoluble, I.09; total, I5.52; available, 14.43 .

5I47. BAKER'S DISSOLVED S. C. BONE. T. P. Licklider, Martinsburg. Guarantee: (As above.) Found: Phos- 
phoric acid, soluble, II.80; reverted, 4.42 ; insoluble, o.86; total, I7.08; available, I6.22.

5034. BAKER'S SPECIAL WHEAT, CORN AND GRASS MIXTURE. Armstrong, Chrislip, Day \& Co., Buckhannon. Guarantee: Phosphoric acid, soluble, 7 ; reverted, 2; insoluble, I ; total, Io; available, 9; nitrogen, o.80; potash, 2; phosphoric acid from dissolved phosphate rock and dissolved animal bone; nitrogen from dissolved fish, tankage and other animal matters; potash from kainit. Found: Phosphoric acid, soluble, 6.70; reverted, 3.60 ; insoluble, I.IO; total, I I.40; available, I0.30 ; nitrogen, I.II; potash, 2.I6; availability of nitrogen, 80 ; chlorine, 2.7 .

5065. CANTON HARROW BRAND CROP GROWER. J. M. Hagerty, Farmington. Guarantee: Phosphoric acid, soluble, 6; reverted, 2 ; insoluble, I ; total, 9; available, 8 ; nitrogen, 0.80 ; potash, I; phosphoric acid from dissolved phosphate rock and dissolved animal matter; nitrogen from dissolved fish, tankage and other animal matter; potash from kainit. Found: Phosphoric acid, soluble, 6.97; reverted, 2.20; insoluble, I.28; total, I0.45; available, 9.I7; nitrogen, I.I7; potash, I.59; availability of nitrogen, 85 ; chlorine, 2.2 .

5160. CANTON EAGLE PHOSPHATE. Washington \& Alexander, Charles Town. Guarantee: Phosphoric acid, soluble, 5 ; reverted, 2 ; insoluble, I ; total, 8 ; available, 7 ; nitrogen, 0.80 ; potash, I; phosphoric acid from dissolved phosphate rock and dissolved animal bone; nitrogen from dissolved fish, tankage and other animal matter; potash from kainit. Found: Phosphoric acid, soluble, 5.46; reverted, 3.25 ; insoluble, I.64; total, I0.35; available, 8.7 I ; nitrogen, I.I 5 ; potash, I.3I ; availability of nitrogen, 74 ; chlorine, 3.2 .

5I63. CANTON SOLUBLE ALKALINE BONE. Washington \& Alexander, Charles Town. Guarantee: Phosphoric acid, soluble, Io; reverted, 2 ; insoluble, I ; total, I3 ; available, I2 ; 
potash, 3; phosphoric acid from dissolved phosphate rock; potash from kainit and muriate of potash. Found: Phosphoric acid, soluble, Io.96; reverted, 3.27 ; insoluble, 0.37 ; total, I4.60; available, I4.23; potash, 2.73 ; chlorine, I.9.

5060. CANTON PATRON'S BONE MIXTURE. Dean \& Reeves, Fairmont. Guarantee: Phosphoric acid, soluble, 8; reverted, 2 ; insoluble, 5 ; total, I 5 ; available, Io; nitrogen, 1.65; potash, 2.50; phosphoric acid available from dissolved phosphate rock and dissolved animal bone; insoluble from undissolved animal bone; nitrogen from dissolved fish, tankage and animal bone; potash from kainit. Found: Phosphoric acid, soluble, 3.84; reverted, 6.I4; insoluble, 4.82 ; total, I4.80; available, 9.98 ; nitrogen, I.74; potash, 2.45 ; availability of nitrogen, 80 ; chlorine, 2.7 .

4975. CLEVELAND DRYER FOREST CITY BUCKEYE. Bishop \& Barbe, Jane Lew. Guarantee: Phosphoric acid, soluble, 7 ; reverted, 2 ; insoluble, I ; total, Io; available, 9 ; nitrogen, 2.47; potash, 2 ; phosphoric acid from dissolved phosphate rock and dissolved animal bone; nitrogen from dissolved fish, tankage and other animal matter; potash from kainit. Found: Phosphoric acid, soluble, 6.86; reverted, 3.62 ; insoluble, 0.97 ; total, I0.45; available, 9.48 ; nitrogen, 2.83 ; potash, 2.75 ; availability of nitrogen, 93 ; chlorine, I.6.

4967. DETRICK'S PARAGON AMMONIATED BONE PHOSPHATE. W. Corrothers, Little Falls. Guarantee: Phosphoric acid, soluble, 5; reverted, 2; insoluble, I ; total, 9; available, 7 ; nitrogen, 0.80 ; potash, I ; phosphoric acid from dissolved phosphate rock and dissolved animal bone; nitrogen from dissolved fish, tankage and other animal matter; potash from kainit. Found: Phosphoric acid, soluble, 6.55; reverted, 3.II ; insoluble, 1.25 ; total, $9.9 \mathrm{I}$; available, 8.66 ; nitrogen, I.I I ; potash, I.07; availability of nitrogen, 78 ; chlorine, 6.4 .

Remark: Chlorine excessive. 
GROWER. A. E. Alexander, Moatsville. Guarantee: Phosphoric acid, soluble, 6 ; reverted, 2 ; insoluble, I ; total, 9 ; available, 8 ; nitrogen, 0.8235 ; potash, 2 ; phosphoric acid from dissolved phosphate rock and dissolved animal bone; nitrogen from dissolved fish, tankage and other animal matter; potash from kainit. Found: Phosphoric acid, soluble, 5.55; reverted, 3.37 ; insoluble, 0.67 ; total, 9.59 ; available, 8.92 ; nitrogen, 0.94 ; potash, 2.19; availability of nitrogen, 87 ; chlorine, 5 .

Remark: Chlorine excessive.

5073. GREAT EASTERN ENGLISH WHEAT GROWER. C. A. Anglin, Philippi. Guarantee: (As above.) Found: Phosphoric acid, soluble, 5.08; reverted, 3.06 ; insoluble, I.06; total, 9.20; available, 8.I4; nitrogen, 0.85; potash, 2.13; availability of nitrogen, 72 ; chlorine, 3 .

5093. GREAT EASTERN ENGLISH WHEAT GROWER. Grape England, Belington. Guarantee: (As above.) Found: Phosphoric acid, soluble, 4.96; reverted, 4.57; insoluble, I.66; total, I I.I9; available, 9.53 ; nitrogen, 0.98; potash, 2.2I : availability of nitrogen. 79; chlorine, 2.7 .

\section{I26. GREAT EASTERN ENGLISH WHEAT} GROWER. George A. Herring, Kingwood. Guarantee: (As above.) Found: Phosphoric acid, soluble, 6.29; reverted, 2.32; insoluble, I.28; total, 9.89; available, 8.6I ; nitrogen, 0.97 ; potash, 2.I I ; availability of nitrogen, 79 ; chlorine, I.9.

\section{I4I GREAT EASTERN ENGLISH WHEAT} GROWER. C. E. Casler, Berkeley Springs. Guarantee: (As above.) Found: Phosphoric acid, soluble, 5.82; reverted, 2.93; insoluble, I.32 ; total, I0.07; available, 8.75 ; nitrogen, 0.95 ; potash, 2.20 ; availability of nitrogen, 80 ; chlorine, 2.5 .

5072. GREAT EASTERN CORN FERTILIZER. C. A. Anglin, Philippi. Guarantee: Phosphoric acid, soluble, 6; reverted, 2 ; insoluble, I ; total, 9; available, 8; nitrogen, 0.8235 ; 
potash, 4 ; phosphoric acid from dissolved phosphate rock and dissolved animal bond; nitrogen from dissolved fish, tankage and other animal matter; potash from kainit and muriate of potash. Found: Phosphoric acid, soluble, 4.96; reverted, 3.37 ; insoluble, I ; total, 9.33 ; available, 8.33 ; nitrogen, 0.97 ; potash, 3.92 ; availability of nitrogen, 77 ; chlorine, 2.3 .

5I40. GREAT EASTERN CORN FERTILIZER. C. E. Casley, Berkeley Springs. Guarantee: (As above.) Found: Phosphoric acid, soluble, 4.04; reverted, 3.6I ; insoluble, I.80; total, 9.45 ; available, 7.65 ; nitrogen, 1.04 ; potash, 4.07 ; availability of nitrogen, 86 ; chlorine, 2.I.

Remark: Available phosphoric acid low.

5083. GREAT EASTERN SOLUBLE BONE AND POTASH. C. A. Anglin, Philippi. Guarantee: Phosphoric acid, soluble, 8 ; reverted, 2 ; insoluble, I ; total, II ; available, Io ; potash, 2; phosphoric acid from dissolved phosphate rock; potash from kainit. Found: Phosphoric acid, soluble, 6.9I ; reverted, 3.I4; insoluble, 0.67 ; total, I0.72; available, I0.05; potash, I.93; chlorine, 5.I.

Remark: Chlorine excessive.

5092. GREAT EASTERN SOLUBLE BONE AND POTASH. Grape England, Belington. Guarantee: (As above.) Found: Phosphoric acid, soluble, 7.85 ; reverted, 2.72 ; insoluble, 0.75 ; total, II.32 ; a vailable, I0.57 ; potash, 2.15 ; chlorine, 6.5 .

Remark: Chlorine excessive.

5082. GREAT EASTERN DISSOLVED BONE. C. A. Anglin, Philippi. Guarantec: Phosphoric acid, soluble, I2; reverted, 2 ; insoluble, I ; total, I 5 ; available, I4; phosphoric acid from dissolved phosphate rock. Found: Phosphoric acid, soluble, Io.08; reverted, 5.24 ; insoluble, 0.63 ; total, 15.95 ; available, I $5 \cdot 32$.

5039. LAZARETTO CROP GROWER. T. B. Drum- 
mond, Buckhannon. Guarantee: Phosphoric acid, soluble, 6; reverted, 2 ; insoluble, I ; total, 9 ; available, 8 ; nitrogen, I.65; potash, 2 ; phosphoric acid from dissolved phosphate rock and dissolved animal bone; nitrogen from fish, bone, tankage and other animal matter; potash from kainit. Found: Phosphoric acid. soluble, 5.19; reverted, 3.62 ; insoluble, 0.73; total, 9.54; available, 8.81; nitrogen, I.72; potash, I.96; availability of nitrogen, 86; chlorine, 3.9.

504I. LAZARETTO SPECIAL POTATO AND TOBACCO FERTILIZER. T. B. Drummond, Buckhannon. Guarantee: Phosphoric acid, soluble, 6; reverted, 2 ; insoluble, I ; total, 9; available, 8 ; nitrogen, 0.82 ; potash, 4; phosphoric acid from dissolved phosphate rock and dissolved animal bone; nitrogen from fish, bone, tankage and other animal matter; potash from manure salts and kainit. Found: Phosphoric acid, soluble, 2.96; reverted, $5: 23$; insoluble, 0.79; total, 8.98; available, 8.19; nitrogen, I.24; potash, 3.90 ; availability of nitrogen, 70; chlorine, 2.7 .

5069. LAZARETTO HIGH GRADE DISSOLVED $B O N E$ AND POTASH. A. G. Chrislip, Philippi. Guarantee: Phosphoric acid, soluble, IO; reverted, 2 ; insoluble, I ; total, I3; available, I2 ; potash, 5 ; phosphoric acid from dissolved phosphate rock; potash from kainit and muriate of potash. Found: Phosphoric acid, soluble, 9.67 ; reverted, 2.85; insoluble, 0.23 ; total, I2.75; available, I2.52; potash, 4.67 ; chlorine, I.9.

Remark: Potash low.

4948. MARYLAND AMMONIATED BONE. J. L. Woodyard, Pruntytown. Guarantee: Phosphoric acid, soluble, 6; reverted, 2 ; insoluble, I ; total, 9 ; available, 8 ; nitrogen, I.65; potash, 3 ; phosphoric acid from dissolved phosphate rock and dissolved animal bone; nitrogen from dissolved fish, tankage and other animal matter; potash from kainit and muriate of potash. Found: Phosphoric acid, soluble, 5.67; reverted, 3.33; insoluble, 
0.99; total, 9.99; available, 9; nitrogen, I.I4; potash, 2.92 ; availability of nitrogen, 84 ; chlorine, $3 \cdot 3$.

Remark: Nitrogen low.

5088. MARYLAND AMMONIATED BONE. J. L. Woodyard, Pruntytown. Guarantee: (As above.) Found: Phosphoric acid, soluble, 2.90; reverted, 5.33; insoluble, 3.28: total, II.5I ; available, 8.23 ; nitrogen, I.93; potash, 3.32 ; availability of nitrogen, 88 ; chlorine, I.5.

4949. MARYLAND TORNADO FERTILIZER. J. L. Woodyard, Pruntytown. Guarantee: Phosphoric acid, soluble, IO; reverted, 2 ; insoluble, I ; total, I3 3 available, I2 ; potash, 5 ; phosphoric acid from dissolved phosphate rock; potash from kainit and muriate of potash. Found: Phosphoric acid, soluble, I0.76; reverted, I.43; insoluble, 0.36 ; total, I2.55; available, I2.19; potash, 5.48 ; chlorine, I.I.

5089. MARYLAND TORNADO FERTILIZER...J. L. Woodyard, Pruntytown. Guarantee: (As above.) Found: Phosphoric acid, soluble, 9.21; reverted, 3.46; insoluble, I.I5; total, I3.82; available, I 2.67 ; potash, 4.97 : chlorine, I.2.

4950. MARYLAND BONO SUPER PHOSPHATE. J. L. Woodyard, Pruntytown. Guarantec: Phosphoric acid, soluble, 8 ; reverted, 2 ; insoluble, I ; total, I I ; available, Io ; potash, 2 : phosphoric acid from dissolved phosphate rock; potash from kainit. Found: Phosphoric acid, soluble, 8.19; reverted, 2.I I ; insoluble, 0.56 ; total, I0.86; available, I0.30; potash, 2.12 ; chlorine, 4.7 .

Remark: Chlorine excessive.

495I. MARYLAND BONO SUPER PHOSPHATE. J. W. Minear, Parsons. Guarantee: (As above.) Found: Phosphoric acid, soluble, 5.90; reverted, 4.46; insoluble, 0.45 ; total, IO.8I ; available, I0.36; potash, 2.I4 ; chlorine, 3.4 . 
5090. BONO SUPER PHOSPHATE. J. L. Woodyard, Pruntytown. Guarantee: (As above.) Found: Phosphoric acid, soluble, 7.I3; reverted, 3.42 ; insoluble, I.Io; total, I I.65: available, I0.55; potash, I.88; chlorine, 3.3 .

5087. MARYLAND DISSOLVED S. C. BONE. J. L. Woodyard, Pruntytown. Guarantee: Phosphoric acid, soluble, I2 ; reverted, 2 ; insoluble, I ; total, I5 ; available, I4; phosphoric acid from dissolved phosphate rock. Found: Phosphoric acid, soluble, I2.1o; reverted, 3.79 ; insoluble, 0.87 ; total, I6.76; available, I 5.89.

5I7I. MARYLAND DISSOLVED S. C. BONE. G. T. Hodges, Shepherdstown. Guarantee: (As above.) Found: Phosphoric acid, soluble, I0.94; reverted, 4.87 ; insoluble, I.42; total, I7.23; available, I5.8I.

5III. REESE'S HIGH GRADE POTASH MIXTURE. W. F. Sill, Pennsboro. Guarantee: Phosphoric acid, soluble, Io; reverted, 2 ; insoluble, I ; total, I3 ; available, I2 ; potash, 5 ; phosphoric acid from dissolved phosphate rock; potash from kainit and muriate of potash. Found: Phosphoric acid, soluble, I0.02; reverted, 3.04 ; insoluble, 0.32 ; total, I3.38; available, I3.06; potash, $5.2 \mathrm{I}$; chlorine, I.7.

4987. SUSQUEHANNA SUPERIOR ROCK PHOSPHATE. J. H. Miller \& Son, Martinsburg. Guarantee: Phosphoric acid, soluble, I2 ; reverted, 2 ; insoluble, I; total, I5; available, I4; phosphoric acid from dissolved phosphate rock. Found: Phosphoric acid, soluble, 8.48; reverted, 7.05; insoluble, 0.92 ; total, I6.45; available, I 5.53 .

5I44. SUSQUEHANNA SUPERIOR ROCK PHOSPHATE. — Adams, Keyser. Guarantee: (As above.) Found: Phosphoric acid, soluble, I2; reverted, 4.I2; insoluble, 0.64; total, І6.76; available, г6.12.

5I78. SUSQUEHANNA SUPERIOR ROCK PHOSPHATE. J. H. Miller \& Son, Martinsburg. Guarantee: (As above.) Found: Phosphoric acid, soluble, I2.70; reverted, 3.99; insoluble, 0.64 ; total, I7.33; available, I6.69. 
4988. SUSQUEHANNA XXV PHOSPHATE. I. H. Miller \& Son, Martinsburg. Guarantec: Phosphoric acid, soluble, 6; reverted, 2; insoluble, I; total, 9; available, 8; nitrogen, o.8o; potash. I; phosphoric acid from dissolved phosphate rock and dissolved animal bone; potash from kainit; nitrogen from dissolved fish, tankage and other animal matter. Found: Phosphoric acid, soluble, 6.56; reverted, I.98; insoluble, I.36; total, 9.90 : available, 8.54 ; nitrogen, 1.06 ; potash, I.I 4 ; availability of nitrogen, 83 ; chlorine, 2.6 .

5166. SUSQUEHANNA XXV PHOSPHATE. W. N. Lemen \& Son, Shepherdstown. Guarantee: (As above.) Fonnd: Phosphoric acid, soluble, 4.66; reverted, 4.I2; insoluble, I.7I ; total, I0.49; available, 8.78; nitrogen, 0.99; potash, I.30; availability of nitrogen, 82 ; chlorine, I0.7.

5020. SUSQUEHANNA POTATO AND TOBACCO PHOSPHATE. J. H. Miller \& Son, Martinsburg. Guarantec: Phosphoric acid, soluble, 6; reverted, 2 ; insoluble, I; total, 9; available, 8 ; nitrogen, I.65; potash, 5 ; phosphoric acid from dissolved phosphate rock and dissolved animal bone; nitrogen from fish, bone, tankage and other animal matter; potash from muriate of potash and kainit. Found: Phosphoric acid, soluble, 4.82; reverted, 3.96; insoluble, 0.79; total, 9.57 ; available, 8.78; nitrogen, I.95; potash, 5.66 ; availability of nitrogen, 83 ; chlorine, 2.

4969. WASHINGTON \& ALEXANDER'S REGULAR CORN FERTILIZER. Washington \& Alexander, Charles Town. Guarantce: Phosphoric acid, soluble, 6; reverted, 2; insoluble, I; total, 9; available, 8; potash, 5; phosphoric acid from dissolved phosphate rock; potash from muriate of potash and kainit. Found: Phosphoric acid, soluble, 6.79; reverted, 2.8I ; insoluble, o.82 ; total, 9.42; available, 8.60; potash, 5.49: chlorine, I.3.

50I2. WILLIAMS \& CLARK'S ROYAL BONE PHOSPHATE. O. R. Carmen, Wellsburg. Guarantee: Phosphoric 
acid, soluble, 6; reverted, 2 ; insoluble, I ; total, 9; available, 8 ; nitrogen, 1.03; potash, 2 ; phosphoric acid from dissolved phosphate rock and dissolved animal bone; nitrogen from dissolved fish, tankage and other animal matter; potash from kainit. Found: Phosphoric acid, soluble, 4.70; reverted, 3.96; insoluble, I.78; total, I0.44; available, 8.66; nitrogen, I.02; potash, 2.59; availability of nitrogen, 90 ; chlorine, 2.2 .

5067. WILLIAMS \& CLARK'S ACORN ACID PHOSPHATE. A. G. Chrislip, Philippi. Guarantee: Phosphoric acid, soluble, I2 ; reverted, 2 ; insoluble, I ; total, I5; available, I4; phosphoric acid from dissolved phosphate rock. Found: Phosphoric acid, soluble, I0.04; reverted, 5.93; soluble, 0.87 ; total, I6.84; available, I 5.97 .

5095. WILLIAMS \& CLARK'S ACORN ACID PHOSPHATE. A. G. Chrislip, Philippi. Guarantee: (As above.) Found: Phosphoric acid, soluble, I2.29; reverted, 3.03 ; insoluble, I.46; total, I6.78; available, I 5.32.

5068. WILLIAMS \& CLARK'S DISSOLVED BONE AND POTASH. A. G. Chrislip, Philippi. Guarantee: Phosphoric, acid, soluble, 8 ; reverted, 2 ; insoluble, I ; total, II ; available, Io; potash, 2 ; phosphoric acid from dissolved rock; potash from kainit. Found: Phosphoric acid, soluble, 5.88; reverted, 3.86; insoluble, 0.42 ; total, Io.I6; available, 9.74; potash, 2.27 ; chlorine, 3.7 .

Remark: Available phosphoric acid low.

5097. WILLIAMS \& CLARK'S PROLIFIC CROP PRODUCER. A. G. Chrislip, Philippi. Guarantee: Phosphoric acid, soluble, 5 ; reverted, 2 ; insoluble, I ; total, 8 ; available, 7 ; nitrogen, 0.80 ; potash, I ; phosphoric acid from dissolved phosphate rock and dissolved animal bone; nitrogen from dissolved fish, tankage and other animal matter; potash from kainit. Found. Phosphoric acid, soluble, 5.65; reverted, 2.59; insoluble, r.46; 
total, 9.70 ; available, 8.24 ; nitrogen, I.I3; potash, I.20; availability of nitrogen, 8I ; chlorine, 4.I.

5I28. WILLIAMS \& CLARK'S PROLIFIC CROP PRODUCER. W. R. Dudley, Wheeling. Guarantee: (As above.) Found: Phosphoric acid, soluble, 3.07 ; reverted, 4.02 ; insoluble, I.84; total, 8.93; available, 7.09; nitrogen, 0.93: potash, I.49; availability of nitrogen, 83 ; chlorine, I.9.

4993. ZELL'S ECONOMIZER PHOSPHATE. The Exchange Mill Company, Grafton. Guarantee: Phosphoric acid, soluble, 6; reverted, 2 ; insoluble, I ; total, 9 ; available, 8 ; nitrogen, o.8o; potash, 2; phosphoric acid from dissolved phosphate rock and dissolved animal bone; nitrogen from dissolved fish, tankage and other animal matters; potash from kainit. Found: Phosphoric acid, soluble, 6.72; reverted, 2.48; insoluble, I.I4; total, I0.34; available, 8.20 ; nitrogen, 0.97 ; potash, 2.02 ; availability of nitrogen, 85 ; chlorine, $3 \cdot 3$.

5054. ZELL'S ECONOMIZER PHOSPHATE. J. L. Hall, Fairmont. Guarantee: (As above.) Found: Phosphoric acid, soluble, 4.72 ; reverted, 3.70 ; insoluble, 0.96 ; total, 9.38 ; available, 8.42 ; nitrogen, 0.94 ; potash, 2.50 ; availability of nitrogen, 74 ; chlorine, 4.

5IOO. ZELL'S ECONOMIZER PHOSPHATE. The Exchange Mill Company, Grafton. Guarantec: (As above.) Found: Phosphoric acid, soluble, 3.28; reverted, 5.22; insoluble, 2.02 ; total, I0.52; available, 8.50 ; nitrogen, I.I I ; potash, I.98 . availability of nitrogen, $8 \mathrm{I}$; chlorine, 2.9 .

4995. ZELL'S ELECTRIC PHOSPHATE. Exchange Mill Co., Grafton. Guarantee: Phosphoric acid, soluble, 8; reverted, 2 ; insoluble, I ; total, II ; available, IO ; potash, 2 ; phosphoric acid from dissolved phosphate rock; potash from kainit. Found: Phosphoric acid, soluble, 5.7 I ; reverted, 4.41 insoluble, 0.42 ; total, I0.54; available, IO.I2; potash, 2.08 ; chlorine, 3.7 . 
5056. ZELL'S ELECTRIC PHOSPHATE. J. L. Hall, Fairmont. Guarantee: (As above.) Found: Phosphoric acid, soluble, 5.27; reverted, 5.08; insoluble, I.36; total, II.7I ; available, I0.35; potash, 2.27 ; chlorine, 4.9 .

Remark: Chlorine excessive.

5102. ZELL'S ELECTRIC PHOSPHATE. Exchange Mill Co., Grafton. Guarantee: (As above.) Found: Phosphoric acid, soluble, 5.86; reverted, 4.75 ; insoluble, I.06; total, I . 67 ; available, Iо.6 ; potash, 2.12 ; chlorine, 3 .

5I82. ZELL'S ELECTRIC PHOSPHATE. R. Hunter, Berkeley Springs. Guarantee: (As above.) Found: Phosphoric acid, soluble, 7.66; reverted, 3.75 ; insoluble, 0.77 ; total, I2.I8; available, II.4I ; potash, 2.1 I ; chlorine, 2.9.

4994. ZELL'S DISSOLVED BONE PHOSPHATE. The Exchange Mill Co., Grafton. Guarantee: Phosphoric acid, soluble, I2 ; reverted, 2 ; insoluble, I ; total, I 5 ; available, I4 ; phosphoric acid from dissolved phosphate rock. Found: Phosphoric acid, soluble, 8.32 ; reverted, 7.74 ; insoluble, 0.79; total, I6.85; available, $\mathbf{6 . 0 6 . ~}$

5094. ZELL'S DISSOLVED BONE PHOSPHATE. Philippi Mill Co., Philippi. Guarantee: (As above.) Found: Phosphoric acid, soluble, 9.08; reverted, 6.59; insoluble, I.OI; total, I6.68; available, I 5.67.

5IOI. ZELL'S DISSOLVED BONE PHOSPHATE. The Exchange Mill Co., Grafton. Guarantee: (As above.) Found: Phosphoric acid, soluble, I2.6I ; reverted, 3.38; insoluble, o.9o; total, I6.89; available, I 5.99 .

5183. ZELL'S DISSOLVED BONE PHOSPHATE. R. Hunter, Berkeley Springs. Guarantee: (As above.) Found: Phosphoric acid, soluble, I2.5I ; reverted, 3.86; insoluble, o.8I ; total, I7.I8; available, I6.37. 
502I. ZELL'S LITTLE GIANT. Cicero Philips, Beling ton. Guarantee: Phosphoric acid, soluble, 5; reverted, 2 ; insoluble, I ; total, 8 ; available, 7 ; nitrogen, 0.80 ; potash, I ; phosphoric acid from dissolved phosphate rock and dissolved animal bone; nitrogen from dissolved fish, tankage and other animal matter; potash from kainit. Found: Phosphoric acid, soluble, 6.51; reverted, 2.06; insoluble, I.47; total, I0.04; available, 8.57 : nitrogen, I.20; potash, I.38; availibility of nitrogen, 82 ; chlorine, $5 \cdot 3$.

Remark: Chlorine excessive.

5055. ZELL'S LITTLE GIANT. J. L. Hall, Fairmont. Guarantee: (As above.) Found: Phosphoric acid, soluble, 3.90 ; reverted, 3.50 ; insoluble, I.09; total, 8.49; available, 7.40; nitrogen, I.28; potash, I.57; availability of nitrogen, 8 I ; chlorine, $7 \cdot 5$.

Remark: Chlorine excessive.

5I39. ZELL'S LIT'LLE GIANT. R. Hunter, Berkeley Springs. Guarantee: (As above.) Found: Phosphoric acid, soluble, 5.32 ; reverted, 2.94 ; insoluble, I.4I ; total, 9.67 ; available, 8.26 ; nitrogen, I.07; potash, I.24; availability of nitrogen, 75 ; chlorine, 3.7 .

\section{THE ARMOUR FERTILIZER WORKS,}

BALTIMORE, MD.

50I4. HIGH GRADE POTATO. W. H. Bailey Hardware Co., Morgantown. Guarantee: Phosphoric acid, soluble, 6; reverted, 2 ; insoluble, I ; total, 9 ; available, 8 ; nitrogen, I.64; potash, Io; phosphoric acid from acid phosphate and animal bone; nitrogen from tankage, blood and, or nitrate of soda; potash from muriate, sulphate and, or kainit. Found: Phosphoric acid, soluble, 7.89 ; reverted, I.96; insoluble, o.91 ; total, I0.76; available, 9.85; nitrogen, I.80; potash, I0.07; availability of nitrogen, 95 ; chlorine, 0.6 . 
5OI 5. STAR PHOSPHATE. W. H. Bailey Hardware Co., Morgantown. Guarantee: Phosphoric acid, soluble, I2; reverted, 2; insoluble, 2 ; total, I6; available, I4; phosphoric acid from acid phosphate. Found: Phosphoric acid, soluble, I2.56; reverted, 2.77 ; insoluble, 0.37 ; total, I5.70; available, I 5.33 .

5156. STAR PHOSPHATE. R. N. Stewart \& Son, Martinsburg. Guarantee: (As above.) Found: Phosphoric acid, soluble, I2.87 ; reverted, 2.9 I insoluble, 0.57 ; total, I6.35; available, I 5.78 .

50I6. WHEAT, CORN AND OAT SPECIAL. W. H. Bailey Hardware Co., Morgantown. Guarantee: Phosphoric acid, soluble, 5 ; reverted, 2 ; insoluble, I; total, 8 ; available, 7 ; nitrogen, 0.82 ; potash, I; phosphoric acid from acid phosphate and animal bone; nitrogen from tankage, blood and, or nitrate of soda; potash from muriate, sulphate and, or kainit. Found: Phosphoric acid, soluble, 6.I4; reverted, 2.70; insoluble, 0.69: total, 9.53 ; available, 8.84 ; nitrogen, 0.99 ; potash, I.24; availability of nitrogen, 90 ; chlorine, 3.5 .

Remark: Source of potash equivalent to kainit.

5062. WHEAT, CORN AND OAT SPECIAL. J. M. Hagerty, Farmington. Guarantee: (As above.) Found: Phosphoric acid, soluble, 7.46; reverted, i.89; insoluble, 0.19; total, 9.54; available, 9.35 ; nitrogen, 0.87 ; potash, 0.89 ; availability of nitrogen, 91 ; chlorine, 5.2.

Remark: Chlorine excessive.

50I7. ROYAL AMMONIATED BONE. W. H. Bailev Hardware Co., Morgantown. Guarantee: Phosphoric acid, soluble, 6; reverted, 2 ; insoluble, I ; total, 9; available, 8 ; nitrogen, 0.82 ; potash, 4 ; phosphoric acid from acid phosphate and animal bone; nitrogen from tankage, blood and, or nitrate; potash from muriate, sulphate and, or kainit. Found: Phosphoric acid, soluble, 7.61 ; reverted, I.49; insoluble, 0.65; total, 9.75; available, 
9.IO; nitrogen, o.88; potash, 4.I2; availability of nitrogen, 93 ; chlorine, 2.6.

Remark: Potash from kainit.

50I8. BONE MEAL. W. H. Bailey Hardwarè Co., Morgantow1. Guarantee: Phosphoric acid, reverted, ro; insoluble. I4; total, 24 ; available, I0; nitrogen, 2.47 ; phosphoric acid from animal bone; nitrogen from animal bone. Found: Phosphoric acid, total, 24.68 ; nitrogen, 3.03 ; availability of nitrogen, So.

5II3. BONE MEAL. Bishop \& Barbe, Jane Lew. Guarantee: (As above.) Found: Phosphoric acid, total, 24.83; nitrogen, 3.I2; availability of nitrogen, 88.

5064. PHOSPHATE AND POTASH. J. M. Hagerty, Farmington. Guarantee: Phosphoric acid, soluble, 8; reverted, 2 ; insoluble, I ; total, I I ; available, Io; potash, 2 ; phosphoric acid from acid phosphate; potash from muriate, sulphate and, or kainit. Found: Phosphoric acid, soluble, 8.44; reverted, 2.79; insoluble, 0.37; total, II.50; available, II.I3; potash, 2; chlorine, 4.4 .

Remark: Potash from kainit.

5155. PHOSPHATE AND POTASH. R. N. Stewart \& Son, Martinsburg. Guarantee: (As above.) Found: Phosphoric acid, soluble, 8.56; reverted, 2.40; insoluble, 0.69; total. I 1.65 ; available, I0.96; potash, 2.4 I ; chlorine, 3.3 .

Remark: Potash from kainit.

\section{PAUGH \& SONS COMPANY.}

BALTIMORE, MD.

4978. BAUGH'S GENERAL CROP GROWER. T. P. Licklider, Martinsburg. Guarantee: Phosphoric acid, available, 8 ; nitrogen, 0.82 ; potash, I; phosphoric acid from animal bone and phosphate rock; nitrogen from animal bone and tankage; 
potash from muriate of potash and sulphate of potash. Found: Phosphoric acid, soluble, 5.88; reverted, 4.19; insoluble, 2.0I; total, I2.08; available, I0.07 ; nitrogen, I.I 5 ; potash, I.96; availability of nitrogen, 84 ; chlorine, 4.I.

Remark: Potash from kainit, contrary to guarantee.

5038. BAUGH'S GENERAL CROP GROWER. R. T. Lowndes, Clarksburg. Guarantee: (As above.) Found: Phosphoric acid, soluble, 3.6I ; reverted, 6.03; insoluble, 3.91 ; total, I3.55; available, 9.64 ; nitrogen, I.I9; potash, I.86; availability of nitrogen, 82 ; chlorine, 2.8.

Remark: Potash from kainit, contrary to guarantee.

5I35. BAUGH'S GENERAL CROP GROWER. Conaway \& Clayton, Barracksville. Guarantee: (As above.) Found: Phosphoric acid, soluble, 5.14; reverted, 4.23; insoluble, I.79; total, II.I6; available, 9.37 ; nitrogen, 0.93; potash, I.56; availability of nitrogen, 82 ; chlorine, 4.2 .

Remark: Potash from kainit, contrary to guarantee.

5I73. BAUGH'S GENERAL CROP GROWER. T. P. Licklider, Martinsburg. Guarantee: (As above.) Found: Phosphoric acid, soluble, 4.06; reverted, 5.50; insoluble, 2.52; total, I2.08; available, 9.56 ; nitrogen, г..12; potash, 1.47 ; availability of nitrogen, 66 ; chlorine, $3 \cdot 3$.

Remark: Potash from kainit, contrary to guarantee; availability of nitrogen low.

4979. BAUGH'S EXPORT BONE WITH POTASH. T. P. Licklider, Martinsburg. Guarantee: Phosphoric acid, II ; nitrogen, 1.65 ; potash, 2 ; phosphoric acid from animal bone: nitrogen from animal bone; potash from sulphate of potash and muriate of potash. Found: Phosphoric acid, soluble, 0.52; reverted, IO.I3 insoluble, 2.46 ; total, I3.I I ; available, Io.65; nitrogen, I.96; potash, 2.38; availability of nitrogen, 87 ; chlorine, 4.8.

Remark: Potash from kainit, contrary to guarantee. 
498I. BAUGH'S HIGH GRADE ACID PHOSPHATE. T. P. Licklider, Martinsburg. Guarantee: Phosphoric acid, available, I4; phosphoric acid from phosphate rock. Found: Phosphoric acid, soluble, I3.II ; reverted, 3.32 ; insoluble, I.52; total, I7.95; available, I6.43.

5136. BAUGH'S HIGH GRADE ACID PHOSPHATE. Conaway \& Clayton, Barracksville. Guarantec: (As above.) Found: Phosphoric acid, soluble, I3.I4; reverted, 3.57 ; insoluble, 0.4I ; total, I7.I2; available, I6.7 I.

5I76. BAUGH'S HIGH GRADE ACID PHOSPHATE. Phillips Bros., Berkeley Springs. Guarante: (As above.) Found: Phosphoric acid, soluble, I3.45; reverted, 3.38 ; insoluble, 0.70; total, I7.53; available, I6.83.

5022. BAUGH'S DOUBLE EAGLE PHOSPHATE. R. T. Lowndes, Clarksburg. Guarantee: Phosphoric acid, available, 8 ; nitrogen, I.65; potash, I; phosphoric acid from animal bone and phosphate rock; nitrogen from animal bone and tankage; potash from sulphate of potash and muriate of potash. Found: Phosphoric acid, soluble, 2.85 ; reverted, 6.12; insoluble, 3.76 ; total, I2.73; available, 8.97 ; nitrogen, I.74; potash, I.40; availability of nitrogen, 89 ; chlorine, 2.4 .

Remark: Source of potash equivalent to kainit or low-grade muriate.

5138. BAUGH'S DOUBLE EAGLE PHOSPHATE. Dean \& Reeves, Fairmont. Guarantee: (As above.) Found. Phosphoric acid, soluble, 5.80; reverted, 3.03; insoluble, I.89; total, I0.72; available, 8.83 ; nitrogen, I.95; potash, I.76; availability of nitrogen, $\delta_{3}$; chlorine, $3 \cdot 3$.

Remark: Potash from kainit, contrary to guarantee.

5I75. BAUGH'S DOUBLE EAGLE PHOSPHAT'E. Conaway \& Clayton, Barracksville. Guarantec: (As above.) Found: Phosphoric acid, soluble, 5.86: reverted, 3.48; insoluble, 0.92 : 
total, I0.26; available, 9.34; nitrogen, 2.08; potash, I.55; availability of nitrogen, 90 ; chlorine, 3.9 .

Remark: Potash from kainit, contrary to guarantee.

\section{BAUGH'S DOUBLE EAGLE PHOSPHATE.} Dean \& Reeves, Fairmont. Guarantee: (As above.) Found: Phosphoric acid, soluble, 5.88; reverted, 3.02; insoluble, 2.4I ; total, II.3I ; available, 8.90 ; nitrogen, I.94; potash, I.66; availability of nitrogen, 92 ; chlorine, 3.7 .

Remark: Potash from kainit, contrary to guarantee.

5023. BAUGH'S ANIMAL BONE AND POTASH COMPOUND. R. T. Lowndes, Clarksburg. Guarantee: Phosphoric acid ; available, 8 ; nitrogen, I.65; potash, 2 ; phosphoric acid from animal bone and phosphate rock; nitrogen from animal bone and tankage; potash from sulphate of potash and muriate of potash. Found: Phosphoric acid, soluble, 4.48; reverted, 4.54; insoluble, 2.33; total, II.35; available, 9.02; nitrogen, I.74; potash, 2.59; availability of nitrogen, 90 ; chlorine, 3.3 .

Remark: Potash from kainit, contrary to guarantee.

5137. BAUGH'S ANIMAL, BONE AND POT ASH COMPOUND. Conaway \& Clayton, Barracksville. Guarantee: (As above.) Found: Phosphoric acid, soluble, 6.37; reverted, 2.50; insoluble, I ; total, 9.87 ; available, 8.87 ; nitrogen, I.97; potash, 2.37; availability of nitrogen, 73 ; chlorine, 4.2.

Remark: Potash from kainit, contrary to guarantee.

5037. BAUGH'S CORN FERTILIZER. R. T. Lowndes, Clarksburg. Guarantee: Phosphoric acid, available, 8; nitrogen, 0.82 ; potash, 4 ; phosphoric acid from animal bone and phosphate rock; nitrogen from animal bone and tankage; potash from muriate of potash and sulphate of potash. Found: Phosphoric acid, soluble, 2.43; reverted, 5.68; insoluble, 2.44; total, I0.55; available, 8. II ; nitrogen, I.II ; potash, 4.83 ; availability of nitrogen, 85 ; chlorine, 1.8 .

Remark: Source of potash equivalent to low-grade muriate. 
5120. BAUGH'S WHEAT FERTILIZER. R. 'T. Lowndes, Clarksburg. Guarantec: Phosphoric acid, available, 8 ; nitrogen, I.65; potash, 2 ; phosphoric acid from animal bone and phosphate rock; nitrogen from animal bone and tankage; potash from sulphate of potash and muriate of potash. Found: Phosphoric acid, soluble, 5.78; reverted, 2.92; insoluble, 2.60; total, II.30; available, 8.70; nitrogen, 2.I I ; potash, 2.29; availability of nitrogen, 79 ; chlorine, 2.2.

Remark: Source of potash equivalent to low-grade muriate.

5I74. BAUGH'S WHEAT FERTILIZER. Conaway \& Clayton, Barracksville. Guarantee: (As above.) Found: Phosphoric acid, soluble, 6.74; reverted, 2.09; insoluble, 0.82; total, 9.65 ; available, 8.83 ; nitrogen, 1.97 ; potash, 2.50 ; availability of nitrogen, 78 ; chlorine, 3.9 .

Remark: Potash from kainit, contrary to guarantee.

512I. BAUGH'S PURE RAW BONE MEAL. R. T. Lowndes, Clarksburg. Guarantee: Phosphoric acid, 2 I ; nitrogen, 3.70 ; phosphoric acid from animal bone; nitrogen from animal bone. Found: Phosphoric acid, total, 21.07; nitrogen, 3.75 ; availability of nitrógen, 75 .

5177. BAUGH'S PURE RAW BONE MEAL. Conaway \& Clayton, Barracksville. Guarantee: (As above.) Found: Phosphoric acid, total, 2г.86; nitrogen, 3.79; availability of nitrogen, 76.

5146. BAUGH'S SPECIAL POTATO IO PER CENT. POTASH. T. P. Licklider, Martinsburg. Guarantee: Phosphoric acid, available, 5 ; nitrogen, 1.65 ; potash, Io; phosphoric acid from animal bone and phosphate rock; nitrogen from animal bone and tankage; potash from high-grade sulphate of potash. Found: Phosphoric acid, soluble, 3.7I ; reverted, 3.14; insoluble, 2.30 ; total, 9.15; available, 6.85 ; nitrogen, 1.77; potash, I0.16; availability of nitrogen, 9I ; chlorine, 0.4 . 


\section{BALTINORE PULTERIZING COMPANY,}

BALTimore, MD.

5I57. SPECIAL SPRING MIXTURE OR SPECIAL, FALI, MIXTURE. R. N. Stewart \& Son, Nartinsburg. Guarantee: Phosphoric acid, available, 8; potash, I ; phosphoric acid from Florida bone phosphate; potash from sulphate of potash, Found: Phosphoric acid, soluble, I.O7; reverted, 8.33 ; insoluble, I.28; total, Io.68; available, 9.40 ; potash, I.07; chlorine, 2.5 .

Remark: Potash from kainit. contrary to guarantee.

BOIVKER FERTILIZER COMPANY,

Boston. New York and Cincinnati.

503O. BOWKER'S DISSOLVED BONE IVITH POTASH. Dave Stewart. Clarksburg. Guarantee: Phosphoric acid, insoluble. 2 ; total, I2: arailable, Io: potash, 2 : phosphoric acid from phosphate rock: potash from muriate. Found: Phosphoric acid, soluble, 0.95: reverted, 9.3I ; insoluble, 2.62 ; total, I 2.88 : available, I0.26: potash, 2.25 : chlorine. I.

5075. BOWKER'S DISSOLTED BONE WITH POTASH. T. A. MeNear, Lowell. Guarantee: (As above.) Found: Phosphoric acid, soluble. I.06: reverted. 8.83: insoluble, 2.97; total, I2.86; available. 9.89; potash, I.94; chlorine, I.I.

\section{IO9. BOWKER'S DISSOLVED BONE WITH POT-} ASH. W. F. Sill, Pennsboro. Guarantee: (As above.) Found: Phosphoric acid, soluble, 3.13; reverted, 7.05; insoluble, 4.II; total, I4.29; available, IO.I8; potash, I.85; chlorine, I.I.

5II7. BOWKER'S DISSOLVED BONE WITH POT$A S H$. T. B. Drummond, Buckhannon. Guarantee: (As above.) Fonnd: Phosphoric acid, soluble, 2.70; reverted. 7.29; insoluble. 3.70 ; total, I3.69; available, 9.99 ; potash, 2 ; chlorine, I.I. 
5078. BOWKER'S POTASH FERTILIZER. T. A. McNear, Lowell. Guarantee: Phosphoric acid, insoluble, 2 ; total, I2 ; available, Io; potash, 5 ; phosphoric acid from phosphate rock; potash from muriate. Found: Phosphoric acid, soluble, I.36; reverted, 8.68 ; insoluble, 3.65 ; total, I3.69; available, I0.04; pot.. ash, 5.20 ; chlorine, I.

5IO7. BOWKER'S POTASH FERTILIZER. W. F. Sill, Pennsboro. Guarantee: (As above.) Found: Phosphoric acid, soluble, 0.92 ; reverted, 9.I6 insoluble, 3.35 ; total, I3.43; available, I0.08; potash, 5.30 ; chlorine, o.9.

5II8. BOWKER'S POTASH FERTILIZER. T. B. Drummond, Buckhannon. Guarantee: (As above.) Found: Phosphoric acid, soluble, 4.43; reverted, 5.99; insoluble, 3.8I ; total, I4.23; available, II.42; potash, 4.82 ; chlorine, I.I.

5079. BOWKER'S HARVEST BONE. T. A. McNear, Lowell. Guarantee: Phosphoric acid, insoluble, I ; total, 9; available, 8 ; nitrogen, 0.82 ; potash, I ; phosphoric acid from animal matter and phosphate rock; nitrogen from tankage and nitrate of soda; potash from muriate. Found: Phosphoric acid, soluble, I.75; reverted, 7.19; insoluble, 3.5 I ; total, I2.45; available, 9.94; nitrogen, I.I6; potash, I.47; availability of nitrogen, 88; chlorine, 2.6.

Remark: Source of potash equivalent to kainit.

5 IO8. BOIVKER'S HARVEST BONE. W. F. Sill, Pennsboro. Guarantee: (As above.) Found: Phosphoric acid, solu. ble, I.73; reverted, 6.85 ; insoluble, 2.97 ; total, I1.55; available, 8.58; nitrogen, 0.97 ; potash, I.09; availability of nitrogen, 7I ; chlorine, 0.9 .

5I I9. BOWKER'S HARVEST BONE. T. B. Drummond, Buckhannon. Guarantee: (As above.) Found: Phosphoric acid, soluble, I.91 ; reverted, 6.76; insoluble, 2.88; total, I I.55; 
available, 8.67 ; nitrogen, I.0\%; potash, I.04; availability of uitrogen, 80 ; chlorine, I.I.

5080. BOWKER'S SOLUBLE BONE. T. A. McNear, Lowell. Guarantee: Phosphoric acid, insoluble, 2; total, I6; available, I4; phosphoric acid from phosphate rock. Found: Phosphoric acid, soluble, 9.15; reverted, 6.04; insoluble, 3.01 ; total, I8.22 ; available, I5.19.

5IIO. BOWKER'S SOLUBLE BONE. W. F. Sill, Pennsboro. Guarantee: (As above.) Found: Phosphoric acid, soluble, Io.67; reverted, 3.27 ; insoluble, 4.70 ; total, I8.64; available, I3.94.

5I 12. BOWKER'S BONE MEAL. W. F. Sill, Pennsboro. Guarantee: Phosphoric acid, total, 20 ; nitrogen, 1.65; phosphoric acid from bone; nitrogen from bone. Found: Phosphoric acid, total, 20.06 ; nitrogen, 2 ; availability of nitrogen, 92 .

\section{MARIET'TA BONE AND PHOSPHATE COMPANY, MARteitTA, OHIo.}

5009. POTATO AND TRUCK SPECIAL. C. T. Braumlick, Wheeling. Guarantee: Phosphoric acid, total, Io; available, 8 ; nitrogen, 3 ; potash, 5 ; phosphoric acid from bone; nitrogen from bone, blood, meat and nitrate of soda; potash from sulphate of potash. Found: Phosphoric acid, soluble, 2.20; reverted, 5.52 ; insoluble, I.96; total, 9.68 ; available, 7.72 ; nitrogen, 2.2I ; potash, 6.33; availability of nitrogen, 94 ; chlorine, I.

Remark: Available phosphoric acid low; nitrogen low; source of potash equivalent to muriate.

\section{G. OBER \& SONS COMPANY,}

\section{BALTIMORE, MD.}

5I54. S. \& S. SPECIAL WHEAT COMPOUND. R. N. Stewart \& Son, Martinsburg. Guarantee: Phosphoric acid, solu- 
ble. 7 : reverted, 2 : insoluble, 2 : total, I I ; available, $9:$ nitrogen, 0.82 : potash, 2: phosphoric acid from high-grade Florida phosphate, bone, tankage and fish; nitrogen from high-grade bone. blood, tankage, fish and nitrate of soda; potash from sulphate of potash. Found: Phosphoric acid, soluble, 7.63; reverted. 2.06; insoluble, I.68; total, I I.37; available, 9.69 ; nitrogen, I.02; potash. 2.24; availability of nitrogen. 85 ; chlorine, 4 .

Remark: Potash from kainit, contrary to guarantee.

4986. OBER'S DISSOLIED BONE PHOSPHATE. R. N. Stewart \& Son, Martinsburg. Guarantee: Phosphoric acid, soluble, I I ; reverted, 3 ; insoluble, 2 ; total, I6 ; available, I4 ; phosphoric acid from high-grade Florida phosphate. Found: Phosphoric acid, soluble, I5.33; reverted, I.89; insoluble, o.I7; total, I7.39; available, 17.22.

5098. OBER'S DISSOLI'ED BONE PHOSPHATE. A. G. Chrislip. Philippi. Guarantee: (As above.) Found: Phosphoric acid, soluble, I4.5I : reverted, 3.0 I : insoluble. 0.57): total. IS.I I ; available, I 7.52.

5I52. OBER'S DISSOLVED BONE PHOSPHATE. R. N. Stewart \& Son, Martinsburg. Guarantec: (As abore.) Found: Phosphoric acid, soluble. I3; reverted, 3.40: insoluble. 0.68 : total, I7.08: available. I6.40.

¡I65. OBER'S DISSOL. ${ }^{\star} E D$ BONE PHOSPHATE. Washington \& Alexander. Charles Town. Guarantee: (As above.) Found: Phosphoric acid, soluble, I3.93; reverted, 3.43; insoluble, 0.64 ; total. I8; available, 17.36.

5096. OBER'S FARMERS MIXTURE. A. G. Chrislip, Philippi. Guarantee: Phosphoric acid, soluble, 7; reverted, 2; insoluble, 2; total, II ; available, 9; nitrogen. 0.82; potash, 2; phosphoric acid from H. G. Florida phosphate, bone, tankage, fish; nitrogen from high-grade bone. blood, tankage and fish; potash from sulphate. Found: Phosphoric acid, soluble, 6.97; 
reverted, 2.95 ; insoluble, I.80; total, I I.72 ; available, 9.92 ; nitrogen, 0.93 ; potash, 2.30 ; availability of nitrogen, 86 ; chlorine, 3.8 .

Remark: Potash from kainit, contrary to guarantee.

Remark: Potash from kainit, contrary to guarantee.

5'34. OBER'S FARMERS MIXTURE. J. J. Chisler, Fairmont. Guarantee: (As above.) Found: Phosphoric acid, soluble, 8.72 ; reverted, 2.28 ; insoluble, 0.56 ; total, I I.56 ; available, I I ; nitrogen, I.03 ; potash, 2.38 ; availability of nitrogen, 90 ; chlorine, 2.5 .

Rcmark: Potash from kainit, contrary to guarantee.

5I6I. OBER'S DISSOLVED BONE PHOSPHATE AND POTASH. Washington \& Alexander, Charles Town. Guarantee: Phosphoric acid, soluble, 8 ; reverted, 2 ; insoluble, 2 ; total, I2; available, IO; potash, 2 ; phosphoric acid from H. G. Florida phosphate; potash from sulphate and muriate of potash. Found: Phosphoric acid, soluble, 8.30; reverted, 4.49; insoluble, I.4I ; total, I4.20; available, I2.79; potash, I.99; chlorine, I.7.

5I64. LANGDON'S MIXTURE. Washington \& Alexander, Charles Town. Guarantee: Phosphoric acid, soluble, 6; reverted, 2 ; insoluble, I ; total, 9; available, 8; nitrogen, I.03; potash, I.25; phosphoric acid from H. G. Florida phosphate, dissolved - and tankage; nitrogen from nitrate of soda, dissolved and tankage; potash from sulphate of potash. Found: Phosphoric acid, soluble, 8.38 ; reverted, 2.42 ; insoluble, I.48; total, I2.28; available, Io.80; nitrogen, 0.83 ; potash, 2.27 ; availability of nitrogen, 92 ; chlorine, I.3.

Remark: Potash from muriate, contrary to guarantee.

\section{PIEDMONT MT. AIRY GUANO COMPANY,}

BALTTMORE, MD.

4996. PIEDMONT SPECIAL POTASH MIXTURE. Cicero Phillips, Belington. Guarantee: Phosphoric acid, insoluble. I ; total, II ; available, Io; potash, 5 ; phosphoric acid from 
dissolved phosphate rock; potash from muriate of potash and kainit. Found: Phosphoric acid, soluble, II.09; reverted, 1.6o; insoluble, 0.42 ; total, I3.I I ; available, I2.69; potash, 5.09; chlorine, 2.5 .

Remark: Potash from kainit.

4997. PIEDMONT FARMER'S HIGH GRADE BONE: $A N D$ POTASH. Cicero Phillips, Belington. Guarantee: Phosphoric acid, insoluble, 2 ; total, I2 ; available, Io; potash, 2 : phosphoric acid from S. C. rock; potash from kainit. Found: Phosphoric acid, soluble, I. $3^{8}$; reverted, $8.5^{8}$; insoluble, 0.50 ; total, I0.46; available, 9.96 ; potash, 2.04 ; chlorine, 3.2 .

509I. PIEDMONT FARMER'S HIGH GRADE BONE AND POTASH. Cicero Phillips, Belington. Guarantec: (As above.) Found: Phosphoric acid, soluble, 6.98; reverted, 2.88; insoluble, 0.43 ; total, I0.29; available, 9.86 ; potash, I.99; chlorine, $3 \cdot 5$.

5II4. PIEDMONT FARMER'S HIGH GRADE BONE AND POTASH. A. P. Russell \& Co., Buckhannon. Guarantee: (As above.) Found: Phosphoric acid, soluble, 7.69; reverted, 2.25 ; insoluble, o.I4 ; total, I0.08; available, 9.94 ; potash, 2.25 ; chlorine, 3.4 .

4998. PIEDMONT PLOW BRAND. Cicero Phillips, Belington. Guarantee: Phosphoric acid, insoluble, 2 ; total, Io; available, 8 ; nitrogen, 1.64 ; potash, 2 ; phosphoric acid from dissolved bone and dissolved phosphate rock; nitrogen from blood and bone tankage; potash from muriate of potash and kainit. Found: Phosphoric acid, soluble, $5.7 \mathrm{I}$; reverted, 4.78 ; insoluble, 0.49; total, I0.98; available, 9.49; nitrogen, I.35; potash, 2.18; availability of nitrogen, 75 ; chlorine, I.4.

4999. PIEDMONT FARMER'S FAVORITE. Cicero Phillips, Belington. Guarantee: Phosphoric acid, insoluble, 2; total, Io; available, 8 ; nitrogen, o.82; potash, 4 ; phosphoric acid 
from dissolved phosphate rock and dissolved bone tankage; nitrogen from blood and bone tankage. Found: Phosphoric acid, soluble, 3.43 ; reverted, 5.25 ; insoluble, 0.38 ; total, 9.06 ; available, 8.68 ; nitrogen, 0.83 ; potash, 4.42 ; availability of nitrogen, 7 I ; chlorine, I.9.

Remark: Source of potash not guaranteed.

5II5. PIEDMONT SOLUBLE BONE AND POTASH. A. P. Russell \& Co., Buckhannon. Guarantee: Phosphoric acid, -...; potash, 2; phosphoric acid from dissolved phosphate rock; potash from muriate of potash and kainit. Found: Phosphoric acid, soluble, 7.01 ; reverted, 2.I2; insoluble, 0.20; total, 9.33; available, 9.13; potash, 2.39 ; chlorine, 3.3 .

Remark: Potash from kainit; phos. acid not guaranteed.

5124. PIEDMONT SOLUBLE BONE AND POTASH. George A. Herring, Kingwood. Guarantee: (As above.) Found: Phosphoric acid, soluble, 5.86; reverted, 2.32 ; insoluble, 0.52 ; total, 8.70 ; available, 8.18 ; potash, I.85; chlorine, 3.I.

Remark: Potash from kainit.

5II6. PIEDMONT POTATO PRODUCER. A. P. Russell \& Co., Buckhannon. Guarantee: Phosphoric acid, insoluble, 2; total, 7 ; available, 5; nitrogen, 2.09; potash, 6; phosphoric acid from dissolved bone and phosphate rock; nitrogen from bone, tankage, nitrate of soda and blood; potash from high-grade muriate of potash. Found: Phosphoric acid, soluble, 4.29; reverted, I.73; insoluble, 0.45 ; total, 6.47 ; available, 6.02; potash. 5.40 ; nitrogen, 2.24 ; availability of nitrogen, 84 ; chlorine, $\mathrm{I}$.

5I22. PIEDMONT SPECIAL WHEAT AND GRASS COMPOUND. George A. Herring, Kingwood. Guarantee: Phosphoric acid, available, 8.50 ; nitrogen, o.67; potash, 3 ; phosphoric acid from bone phosphate; nitrogen from bone, tankage and animal matter; potash from kainit and muriate. Found: Phosphoric :uril, soluble, 7.15 ; reverted, 2.65 ; insoluble, 0.70 ; 
total, I0.50; available, 9.80 ; nitroge11, 0.97 ; potash, 2.51 ; availability of nitrogen, 92 ; chlorine, 2.9.

Remark: Potash low; source of potash kainit.

\section{I23. PIEDMONT PURE RAW BONE MIXTURE.} George A. Herring, Kingwood. Guarantes: Phosphoric acid, insoluble, 4 ; total, I2 ; available, 8 ; nitrogen, I.02; potash, I.50; phosphoric acid from bone tankage; nitrogen from bone tankage; potash from muriate and kainit. Found: Phosphoric acid, soluble, 2.34 ; reverted, 4.32 ; insoluble, 4.02 ; total, Io.68; available, 6.66 ; nitrogen, I ; potash, 2.94 ; availability of nitrogen, 82 ; chlorine, $3 \cdot 5$.

Remark: Available phosphoric acid low; potash from kainit.

5125. PIEDMONT HARVEST KING. George A. Herring, Kingwood. Guarantee: Phosphoric acid, insoluble, 2; total, II ; available, 9; nitrogen, 0.82 ; potash, 2 ; phosphoric acid from dissolved phosphate rock and dissolved bone tankage; nitrogen from blood and bone tankage. Fonnd: Phosphoric acid, soluble, 7.37 : reverted, 2.05 ; insoluble, I.81 ; total, I0.23; available, 9.42 ; nitrogen, I.05; potash, 2.64 ; availability of nitrogen, So; chlorine, 2.4 .

Remark: Source of potash equivalent to kainit.

\section{RASIN MONUMENTAL COMPANY,}

BAITIMORE, MD.

5I53. S. \& S. SPECIAL IVHEAT COMPOUND. R. A. Stewart \& Son, Martinsburg. Guarantee: Phosphoric acid, soluble, 6; reverted, 2 ; insoluble, I.50; total, 9.50; available, 8 ; nitrogen, I.50; potash, 2 ; phosphoric acid from high-grade Tennessee, Charleston and Florida phosphate, bone, tankage and fish: nitrogen from high-grade blood and bone tankage and fish; potash irom high-grade sulphate of potash. Found: Phosphoric acid, soluble, 5.22: reverted, 3 ; insoluble, 1.48; total, 9.70; available, 
8.22; nitrogen, I.77; potash, 2.I4; availability of nitrogen, 9I ; chlorine, I.3.

Remark: Source of potash equivalent to muriate, contrary to guarantee.

4970. RASIN'S ACID PHOSPHATE. C. W. Mayer \& Son, Terra Alta. Guarantee: Phosphoric acid, insoluble, I.5O; total, I 5.50; available, I4; phosphoric acid from Charleston, Florida and Tennessee rocks. Found: Phosphoric acid, soluble, I0.69: reverted, 4.45 ; insoluble, I.25; total, I6.39; available, I5.I4.

5042. RASIN'S ACID PHOSPHATE. George Carskadon, Keyser. Guarantee: (As above.) Found: Phosphoric acid, soluble, II.44: reverted, 3.64 ; insoluble, I.42; total, I6.50; available, 15.08 .

5I43. RASIN'S ACID PHOSPHATE. George Carskadon, Keyser. Guarantee: (As above.) Found: Phosphoric acid, soluble, II.35; reverted, 3.88 ; insoluble, 0.77 ; total, I6; available, 15.23 .

4971. RASIN'S SPECIAL BONE AND POTASH.. C. W. Mayer \& Son, Terra Alta. Guarantee: Phosphoric acid, soluble, 9; reverted, I ; insoluble, I. 50; total, II.50; available, Io; potash, 5 ; phosphoric acid from Charleston, Florida and Tennessee rock, bone and tankage; potash from muriate and manure salts. Found: Phosphoric acid, soluble, 6.03; reverted, 4.57; insoluble, I.23; total, I . 83 ; available, Io.6o; potash, 3.48 ; chlorine, I.

Remark: Potash low.

4972. RASIN'S DISSOLVED BONE. C. W. Mayer \& Son, Terra Alta. Guarantee: Phosphoric acid, soluble, 8; reverted, 2 ; insoluble, I.5O; total, II.50; available, Io; nitrogen, 2 ; phosphoric acid from Charleston, Florida and Tennessee rocks; nitrogen from dissolved bone and tankage. Found: Phosphoric acid, soluble, 8.25 ; reverted, 4.39 ; insoluble, I.74; total, I4.38; available, I2.64; nitrogen, I.7I; availability of nitrogen, 90.

Remark: Nitrogen low. 
4973. RASIN'S BONE AND POTASH FERTILIZER. C. W. Mayer \& Son, Terra Alta. Guarantee: Phosphoric acid, soluble, 9; reverted, I ; insoluble, I.50; total, I I.50; available, Io; potash, 2 ; phosphoric acid from Charleston, Florida and Tennessee rock, bone and tankage; potash from muriate and manure salt. Found: Phosphoric acid, soluble, 5.I8; reverted, 5.86; insoluble, I.23; total, I2.27; available, II.04; potash, 2.07 ; chlorine, 2.5.

Remark: Source of potash equivalent to kainit.

5050. RASIN'S BONE AND POTASH FERTILIZER. George Carskadon, Keyser. Guarantee: (As above.) Found: Phosphoric acid, soluble, 5.99; reverted, 4.99; insoluble, I.38; total, I I.86; available, 10.48; potash, I.86; chlorine, 2.9.

Remark: Source of potash equivalent to kainit.

5I42. SEA WALL SPECIAL. George Carskadon, Keyser. Guarantee: Phosphoric acid, insoluble, I.5O; total, II.50; available, Io; phosphoric acid from Charleston, Florida and Tennessee rock. Found: Phosphoric acid, soluble, 5.33; reverted, 6.88; insoluble, 3.72 ; total, I 5.93 ; available, I2.2I.

505I. SEA WALL SPECIAL. George Carskadon, Keyser. Guarantee: (As above.) Found: Phosphoric acid, soluble, 4.27 : reverted, 7.31 ; insoluble, I.69; total, I 3.27 ; available, I I.58.

\section{SWIFT \& COMPANY,}

\section{Chicago, Illi.}

4983. SWIFT'S PURE SUPER PHOSPHATE. R. N. Stewart \& Son, Martinsburg. Guarantee: Phosphoric acid, insoluble, 4 ; total, I2 ; available, 8 ; nitrogen, I.64; potash, 2 ; phosphoric acid from acidulated rock phosphate and bone, blood and tankage; nitrogen from bone, blood and tankage; potash from muriate. Fonnd: Phosphoric acid, soluble, 4.54; reverted, 4.7 I ; insoluble, I.28; total, I0.53; available, 9.25 ; nitrogen, I.95; potash, I.77; availability of nitrogen, 95 ; chlorine, 0.5 . 
5158. SWIFT'S PURE SUPER PHOSPHATE. R. N. Stewart \& Son, Martinsburg. Guarantee: (As above.) Found: Phosphoric acid, soluble, 4.I6; reverted, 3.76; insoluble, 2.94; total, Io.86; available, 7.92 ; nitrogen, I.7I ; potash, 2.09 ; availability of nitrogen, 92 ; chlorine, 0.6 .

4984. RAW BONE MEAL. R. N. Stewart \& Son, Martinsburg. Guarantee: Phosphoric acid, total, 23; nitrogen, 3.75; phosphoric acid from animal bone; nitrogen from animal bone. Found: Phosphoric acid, total, 23.27 ; nitrogen, 4.14; availability of nitrogen, 79 .

4985. BONE MEAL. R. N. Stewart \& Son, Martinsburg. Guarantee: Phosphoric acid, total, 25 ; nitrogen, 2.50 ; phosphoric acid from animal bone; nitrogen from animal bone. Found: Phosphoric acid, total, 25.73 ; nitrogen, 2.50 ; availability of nitrogen, 90 .

5049. BONE MEAL. George Carskadon, Keyser. Guarantee: (As above.) Found: Phosphoric acid, total, 26.15; nitrogen, 2.35 ; availability of nitrogen, 85 .

Remark: Nitrogen low.

5I70. BONE MEAL. G. T. Hodges, Shepherdstown. Guarantee: (As above.) Found: Phosphoric acid, tota1, 25.96; nitrogen, 2.43 ; availability of nitrogen, 85 .

Remark: Nitrogen low.

5172. COMPLETE FERTILIZER. G. T. Hodges, Shepherdstown. Guarantee: Phosphoric acid, insoluble, 3 ; total, I I ; available, 8 ; nitrogen, I; potash, I ; phosphoric acid from acidulated rock phosphate and bone, blood and tankage; nitrogen from bone, blood and tankage; potash from muriate. Found: Phosphoric acid, soluble, 5.55; reverted, 3.42 ; insoluble, I.92; total, Io.89; available, 8.97 ; nitrogen, I.33; potash, I.35; availability of nitrogen. 8I ; chlorine, o.9. 


\section{SCIENTIFIC FERTILIZER COMPANY,}

PitTisburg, Pa.

5007. SCIENTIFIC POTATO FERTILIZER. Walter Marshall, Wheeling. Guarantee: Phosphoric acid, soluble, 4; reverted, 4 ; insoluble, 2 ; total, Io ; available, 8 ; nitrogen, 2.50 ; potash, 6; phosphoric acid two-fifths from tankage, three-fifths mineral phosphate; nitrogen from tankage and blood; potash, one-half from muriate of potash, one-half from sulphate of potash. Found: Phosphoric acid, soluble, 3.97; reverted, 3.5I ; insoluble, I.37 ; total, 8.85 ; available, 7.48 ; nitrogen, 2.58 ; potash, 5.67 ; availability of nitrogen, 90 ; chlorine, $\mathrm{I}$.

Remark: Available phosphoric acid low.

5132. SCIENTIFIC POTATO FERTILIZER. Walter Marshall, Wheeling. Guarantee: (As above.) Found: Phosphoric acid, soluble, 3.22; reverted, 4.99; insoluble, 2.89; total, I I.IO; available, 9.21 ; nitrogen, 2.85 ; potash, 4.44 ; availability of nitrogen, 92 ; chlorine, I.

Remark: Potash low.

5008. CORN AND GRAIN. Walter Marshall, Wheeling. Guarantee: Phosphoric acid, soluble, 5; reverted, 3; insoluble, I ; total, 9; available, 8 ; nitrogen, I.64 ; potash, 2 ; phosphoric acid, one-third from tankage, two-thirds from mineral phosphate; nitrogen from blood and tankage; potash from sulphate of potash. Found: Phosphoric acid, soluble, 3.68; reverted, r.90; insoluble, I.08; total, 6.65; available, 5.58; nitrogen, I.30; potash, 2.47 ; availability of nitrogen, 90 ; chlorine, 3.I.

Remark: Available phosphoric acid low; nitrogen low; source of potash equivalent to kainit, contrary to guarantee.

513I. CORN AND GRAIN. Walter Marshall, Wheeling. Guarantee: (As above.) Found: Phosphoric acid, soluble, 3.65; reverted, 2.68; insoluble, 2.16; total, 8.49; available, 6.33; nitrogen, I.6I ; potash, 3.04 ; availability of nitrogen, 90 ; chloline, 2.8 . 
Remark: Available phosphoric acid low; source of potash equivalent to kainit, contrary to guarantee.

5I33. PURE RAW BONE MEAL. Walter Marshall, Wheeling. Guarantee: Phosphoric acid, total, 22 ; nitrogen, 3.25 ; phosphoric acid from bone; nitrogen from bone. Found: Phosphoric acid, total, 21.65 ; nitrogen, 3.83 ; abailability of nitrogen, 90.

5OI3. PURE RAW BONE MEAL. Walter Marshall, Wheeling. Guarantee: (As above.) Found: Phosphoric acid, total, 20.74; nitrogen, 3.62 ; availability of nitrogen, 85 .

\section{TUSCARORA FERTILIZER COMPANY,}

Baltimore, MD.

5006. ACID PHOSPHATE. J. H. Miller \& Son, Martinsburg. Guarantee: Phosphoric acid, soluble, I2; reverted, 2; insoluble, 2 ; total, I6; available, I4; phosphoric acid from acid phosphate. Found: Phosphoric acid, soluble, I2.22; reverted. 3.65 ; insoluble, 0.42 ; total, I6.29; available, I 5.87 .

5I49. ACID PHOSPHATE. J. H. Miller \& Son, Martinsburg. Guarantee: (As above.) Found: Phosphoric acid, soluble, I2 ; reverted, 4.I5; insoluble, O.I5; total, I6.30; available, I6.15.

5019. FRUIT AND POTATO. J. H. Miller \& Son, Martinsburg. Guarantee: Phosphoric acid, soluble, 6; reverted, 2 ; insoluble, I ; total, 9; available, 8 ; nitrogen, I.65; potash, Io; phosphoric acid from acid phosphate and dissolved böne; nitrogen from nitrate, blood and, or tankage; potash from muriate, sulphate and, or kainit. Found: Phosphoric acid, soluble, 4.85; reverted, 4.07 ; insoluble, I.28; total, I0.20; available, 8.92 ; nitrogen, I.76; potash, 9.60 ; availability of nitrogen, 80 ; chlorine, I.2.

Remark: Potash low, derived from muriate. 


\section{VIRGINIA-CAROLINA CHEMICAL COMPANY,}

Richmond, VA.

5I48. V.C. C. CO.'S STANDARD BONE AND POTASH. T. P. Licklider, Martinsburg. Guarantee: Phosphoric acid, soluble, 9; reverted, I ; insoluble, I.50; total, II.50; available, Io; potash, 2 ; phosphoric acid from dissolved Florida and 'Tennessee rocks; potash from muriate and manure salts. Found: Phosphoric acid, soluble, 2.92; reverted, 6.8I ; insoluble, I.32; total, I I.05; available, 9.73 ; potash, 2.09 ; chlorine, I.8.

Remark: Available phosphoric acid low.

5I68. V. C. C. CO.'S STANDARD BONE AND POTASH. G. T. Hodges, Shepherdstown. Guarantec: (As above.) Found: Phosphoric acid, soluble, 5.96; reverted, 4.87 ; insoluble, 0.90; tota1, Ir.73; available, Io.83; potash, 2.13 ; chlorine, I.3.

5I67. V. C. C. CO.'S I4 PER CENT. ACID PHOSPHATE. G. T. Hodges, Shepherdstown. Guarantee: Phosphoric acid, insoluble, I.50; total, I 5.50 ; available, I4; phosphoric acid from Charleston, Florida and Tennessee rock. Found Phosphoric acid, soluble, II.97; reverted, 3.72; insoluble, o.83; total, I6.52; available, I 5.69.

5I69. V. C. C. CO.'S SPECIAL COMPOUND FOR WHEAT. G. T. Hodges, Shepherdstown. Guarantee: Phosphoric acid, soluble, 6; reverted, 2 ; insoluble, I.50; total, 9.50; available, 8; nitrogen, I; potash, I; phosphoric acid fron Charleston, Florida and Tennessee rock; nitrogen from blood, fish and tankage; potash from muriate and manure salt. Found: Phosphoric acid, soluble, 5.09; reverted, 4.40; insoluble, I.oI ; total, I0.50; available, 9.49 ; nitrogen, I.I6; potash. I.7I ; availability of nitrogen, 78 ; chlorine, ז.6.

5076. ALLISON \& ADDISON'S STANDARD ACID PHOSPHATE. E. W. McNear, Lowell. Guarantee: Phos- 
phoric acid, soluble, 9; reverted, 3 ; insoluble, I ; total, I3; available, I2; phosphoric acid from phosphate rock. Found: Phosphoric acid, soluble, 6.88; reverted, 7.44; insoluble, I.29; total, I5.6I ; available, I4.32.

5077. ALLISON \& ADDISON'S LITTLE GIANT GRAIN AND GRASS GROWER. E. W. McNear, Lowell. Guarantee: Phosphoric acid, soluble, 6; reverted, 2 ; insoluble, 2 ; total, Io ; available, 8 ; nitrogen, 0.82 ; potash, 2 ; phosphoric acid from phosphate rock and animal bone; nitrogen from fish, animal tankage and cotton seed meal; potash from muriate, sulphate and double manure salt. Found: Phosphoric acid, soluble, 2.72 ; reverted, 6.I I insoluble, I.or ; total, 9.84; available, 8.83; nitrogen, I.O2; potash, I.80; availability of nitrogen, 83 ; chlorine, 2 .

508I. S. W. TRAVERS \& CO.'S DISSOLVED BONE PHOSPHATE. E. IV. McNear, Lowell. Guarantee: Phosphoric acid, soluble, II; reverted, 3 ; insoluble, I; total, I5; available, I4; phosphoric acid from phosphate rock. Found: Phosphoric acid, soluble, IO.I2; reverted, 4.26 ; insoluble, I.80; total, I6.I8; available, 14.38 .

\section{WALKER, STRATMAN \& COMPANY,}

\section{Pittsseurg, Pa.}

4960. MEAT, BLOOD AND BONE WITH POTASH. Bought by J. W. Sans, Little Falls. Guarantee: Phosphoric acid, soluble, 4 ; reverted, 4 ; insoluble, 2 ; total, Io ; available, 8 ; nitrogen, 3.25 ; potash, 8 ; phosphoric acid from bone and animal tankage; nitrogen from blood and animal tankage; potash from sulphate of potash. Found: Phosphoric acid, soluble, 4.09; reverted, 4.I5; insoluble, I.82; total, I0.06; available, 8.24 ; nitrogen, 3.33 ; potash, 8.17 ; availability of nitrogen, 92 ; chlorine, I.

Remark: Source of potash equivalent to high-grade muriate, contrary to guarantee. 
5OIO. MEAT, BLOOD AND BONE WITH POTASH. C. R. Carmen, Wellsburg. Guarantee: (As above.) Fonnd: Phosphoric acid, soluble, 4.02; reverted, 4.04; insoluble, I.84; total, 9.90 ; available, 8.06 ; nitrogen, 3.22 ; potash, 7.92 ; availability of nitrogen, 93 ; chlorine, I.I.

Remark: Source of potash equivalent to high-grade muriate, contrary to guarantee.

496i. POTATO SPECIAL. J. W. Sans, Little Falls. Guarantee: Phosphoric acid, soluble, 4; reverted, 4; insoluble, 2 ; total, Io; available, 8 ; nitrogen, 2.50; potash, 6 ; phosphoric acid, two-fifths from tankage, three-fifths from mineral phosphate; nitrogen from tankage and blood; potash, one-half from muriate of potash, one-half from sulphate of potash. Found: Phosphoric acid, soluble, 3.88 ; reverted, 3.58 ; insoluble, I.68; total, 9.04; available, 7.36 ; nitrogen, 2.75 ; potash, 5.59 ; availability of nitrogen, $9 \mathrm{I}$; chlorine, I.I.

Remark: Available phosphoric acid low; potash low.

4989. POTATO SPECIAL. Thomas Nuzum, Grafton. Guarantee: (As above.) Found: Phosphoric acid, soluble, 4.50; reverted, 3.06; insoluble, 1.74; total, 9.30; available, 7.56; nitrogen, 2.54 ; potash, 4.76 ; availability of nitrogen, 94 ; chlorine, I.I.

Remark: Available phosphoric acid low; potash low.

5029. POTATO SPECIAL. Kane \& Owens Hardware Co., Weston. Guarantee: (As above.) Found: Phosphoric acid, soluble, 3.95 ; reverted, 3.52 ; insoluble, I.24; total, 8.7 I ; available, 7.47 ; nitrogen, 2.70; potash, 5.50; availability of nitrogen, 87 ; chlorine, I.I.

Remark: Available phosphoric acid low; potash low.

4962. POTASH AND BONE PHOSPHATE. J. W. Sans, Little Falls. Guarantee: Phosphoric acid, soluble, 5.50; reverted, 4.50; insoluble, I ; total, II ; available, Io; potash, 6; phosphoric acid from mineral phosphate; potash from muriate of 
potash. Found: Phosphoric acid, soluble, 4.45; reverted, 4.28; insoluble, I.04; total, I0.77; available, 9.73 ; potash, 5.29; chlorine, I.

Remark: Available phosphoric acid low: potash low.

4963. PHOSPHORIC ACID AND POTASH. J. W. Sans, Little Falls. Guarantee: Phosphoric acid, soluble, 5.50; reverted, 4.50 ; insoluble, I ; total, I I ; available, Io ; potash, 2 ; phosphoric acid from mineral phosphate; potash from sulphate of potash. Found: Phosphoric acid, soluble, 4.43 ; reverted, 5.12 ; insoluble, 0.9I ; total, I0.46; available, 9.55 ; potash, I.8I ; chlorine, 0.3 .

Remark: Available phosphoric acid loiv.

5IO5. PHOSPHORIC ACID AND POTASH. Thomas Nuzum, Grafton. Guarantee: (As above.) Found: Phosphoric acid, soluble, 3.44; reverted, 5.34; insoluble, 2.02; total, io.8o ; available, 8.78; potash, I.92; chlorine, 3.2 .

Remark: Potash from kainit, contrary to gtuarantee.

4964. FOUR FOLD. J. W. Sans, Little Falls. Guarantee: Phosphoric acid, soluble, 5; reverted, 3; insoluble, I; total, 9; available. 8 ; nitrogen, I.64; potash, 2 ; phosphoric acid, one-third from tankage, two-thirds from mineral phosphate; nitrogen from blood and tankage; potash from sulphate of potash. Found: Phosphoric acid, soluble, 4.59; reverted, 2.24; insoluble, I.02; total, 7.85 ; available, 6.83 ; nitrogen, I.06; potash, 2.02 ; availability of nitrogen, 87 ; chlorine, 2.8 .

Remark: Available phosphoric acid low; nitrogen low; source of potash equivalent to kainit, contrary to guarantee.

5028. FOUR FOLD. Kane \& Owens Hardware Co., Weston. Guarantee: (As above.) Found: Phosphoric acid, soluble, 3.98; reverted, 2.01 ; insoluble, I.02 ; total, 7.or ; available, 5.99; nitrogen, I.I9; potash, 2.27; availability of nitrogen, 90; chlorine, 3 . 
Remark: Available phosphoric acid low; source of potash equivalent to kainit, contrary to guarantee.

506r. FOUR FOLD. Dean \& Reeves, Fairmont. Guarantee: (As above.) Found: Phosphoric acid, soluble, 4.03; reverted, I.9I; insoluble, I.02 ; total, 6.96; available, 5.94; nitrogen, I.26; potash, 2.42 ; availability of nitrogen, 9I ; chlorine, 2.8.

Remark: Available phosphoric acid low; nitrogen low; source of potash equivalent to kainit, contrary to guarantee.

5104. FOUR FOLD. Thomas Nuzum, Grafton. Guarantee: (As above.) Found: Phosphoric acid, soluble, 5.04; reverted, 2.32 ; insoluble, I.98; total, 9.34 ; available, 7.36 ; nitrogen, I.37; potash, 3.22 ; availability of nitrogen, 92 ; chlorine, 2.7 .

Remark: Available phosphoric acid low; nitrogen low; potash from kainit, contrary to guarantee.

4965. GRAIN KING. J. W. Sans, Little Falls. Guarantee: Phosphoric acid, soluble, 5 ; reverted, 4 ; insoluble, I ; total, Io; available, 9 ; nitrogen, 0.82 ; potash, 4 ; phosphoric acid, onefifth from tankage, four-fifths from mineral phosphate; nitrogen from tankage and blood; potash from muriate of potash. Found: Phosphoric acid, soluble, 5.07; reverted, 2.65: insoluble, I.07; total, 8.79; available, 7.72 ; nitrogen, I.I8; potash, 4.35 : availability of nitrogen, 83 ; chlorine, I.4.

Remark: Available phosphoric acid low.

4992. GRAIN KING. Thomas Nuzum, Grafton. Guarantee: (As above.) Found: Phosphoric acid, soluble, 4.39; reverted, 2.72; insoluble, I.06; total, 8.17; available, 7.I I ; nitrogen. I.2I ; potash. 3.8 I ; availability of nitrogen, 90 : chlorine, I.3.

Remark: Available phosphoric acid low.

5027. GRAIN KING. Kane \& Owens Hardware Co., IVeston. Guarantee: (As above.) Found: Phosphoric acid, soluble, 4.23; reverted, 2.62; insoluble, I.I3; total, 7.98; available, 
6.85; nitrogen, I.I7; potash, 4.I9; availability of nitrogen, 89; chlorine, I.3.

Remark: Available phosphoric acid low.

5I29. GRAIN KING. C. R. Carmen, Wellsburg. Guarantee: (As above.) Found: Phosphoric acid, soluble, 4.08; reverted, 4.02 ; insolubie, I.87 ; total, 9.97 ; available, 8.Io; nitrogen, I ; potash, 4.45 ; availability of nitrogen, 84 ; chlorine, I.7.

Remark: Available phosphoric acid low.

5I50. GRAIN KING. T. P. Licklider, Martinsburg. Guarantee: (As above.) Found: Phosphoric acid, soluble, 3.94; reverted, 3.98; insoluble, 2.5 I ; total, ro.43; available, 7.92 ; nitrogen, 0.99 ; potash, 4.40 ; availability of nitrogen, 89 ; chlorine, $\mathrm{I} \cdot 3$.

Remark: Available phosphoric acid low.

4966. GRAIN MANURE. J. W. Sans, Little Falls. Guarantee: Phosphoric acid, soluble, 5; reverted, 3 ; insoluble, I; total, 9 ; available, 8 ; nitrogen, o.82; potash, 1 ; phosphoric acid, one-third from tankage, two-thirds from mineral phosphate; nitrogen, from blood and tankage; potash from sulphate of potash. Found: Phosphoric acid, soluble, 4.39; reverted, 2.Ir; insoluble, 0.97 ; total, 7.47 ; available, 6.50; nitrogen, I.26; potash, I.72; availability of nitrogen, 9I ; chlorine, 2.9.

Remark: Available phosphoric, acid low; source of potash kainit, contrary to guarantee.

503I. GRAIN MANURE. A. P. Russell \& Co., Buckhannon. Guarantee: (As above.) Found: Phosphoric acid, soluble, 3.28 ; reverted, 2.58; insoluble, 0.75; total, 6.6I ; available, 5.86 ; nitrogen, I.II; potash, I.66; availability of nitrogen, 87 ; chlorine, 2.8 .

Remark: Potash from kainit, contrary to guarantee.

5r5x. GRAIN MANURE. T. P. Licklider, Martinsburg. Guarantee: (As above.) Found: Phosphoric acid, soluble, 
2.99; reverted, 4.40: insoluble, o.82; total, 8.21 ; available, 7.39 : nitrogen, 0.99 ; potash, I.63; availability of nitrogen, 80 ; chlorine. 2.3 .

Remark: Source of potash low-grade muriate or kainit, contrary to guarantee: available phosphoric acid low.

4990. BONE AND MEAT. Thomas Nuzum. Grafton. Guarantce: Phosploric acid, total, I6; nitrogen, 3.25 ; phosphoric acid from bone and tankage; nitrogen from bone and tankage. Found: Phosphoric acid. total, 16.08: nitrogen, 3.28; availability of nitrogen, 9 I.

5130. BONE AND MEAT. C. R. Carmen, Wellsburg. Guarantec: (As above.) Found: Phosphoric acid, total, I6.9I; nitrogen, 3; availability of nitrogen, 92.

Remark: Nitrogen low.

4991. HELP MATE. Thomas Nuzum, Grafton, II. Va. Guarantee: Phosphoric acid, soluble, 7 ; reverted, 5 ; insoluble, I : total, I 3: arailable, I2; phosphoric acid from mineral phosphate. Found: Phosphoric acid, soluble, o.68; reverted, 7.93 ; insoluble. I.jI ; total, IO.I2; available, 8.6 I.

Remark: Arailable phosphoric acid low.

5032. HELP MATE. A. P. Russell \& Co., Buckhannon. Guarantec: (As above.) Found: Phosphoric acid, soluble. 4.84 ; reverted, 4.69; insoluble. I.57; total, I1.10; available, 9.53.

Remark: Available phosphoric acid low.

5106. HELP MATE. Thomas Nuzum, Grafton. Guarantee: (As above.) Found: Phosphoric acid, soluble, 3.or ; reverted, 8 ; insoluble, $2.8 \mathrm{I}$; total, 13.82 : available. I I.or.

Remark: Available phosphoric acid low.

jISO. HELP MATE. C. R. Carmen, Wellsburg. Guar- 
antee: (As above.) Found: Phosphoric acid, soluble, 5.I6; reverted, 5.90 ; insoluble, 2.55 ; total, I3.61 ; available, I I.o6.

Remark: Available phosphoric acid low.

5002. PURE RAW BONE MEAL. Thomas Nuzum, Grafton. Guarantee: Phosphoric acid, tota1, 22 ; nitrogen, 3.25 ; phosphoric acid from bone; nitrogen from bone. Found: Phosphoric acid, total, 21.30 ; nitrogen, 3.82 ; availability of nitrogen, 90 .

5IO3. PURE RAW BONE MEAL. Thomas Nuzum, Grafton. Guarantee: (As above.) Found: Phosphoric acid, total, 22.46; nitrogen, 3.5I ; availability of nitrogen, 88 .

5I79. PURE RAW BONE MEAL. C. R. Carmen, Wellsburg. Guarantee: (As above.) Found: Phosphoric acid, total, $2 \mathrm{I} .53$; nitrogen, 3.74 ; availability of nitrogen, 89 .

518I. PURE RAW BONE MEAL. George B. Shank, Keyser. Guarantee: (As above.) Found: Phosphoric acid, total, 23.46; nitrogen, 3.99; availability of nitrogen, 87 .

ROBERT A. WOOLDRIDGE COMPANY, BALTTMORE, MD.

4953. FLORIDA ACID PHOSPHATE. J. W. Minear, Parsons. Guarantee: Phosphoric acid, available, I4; phosphoric acid from dissolved rock. Found: Phosphoric acid, soluble, I I.28; reverted, 3.10 ; insoluble, I.55; total, I 5.93 ; available, I4.38.

5004. FLORIDA ACID PHOSPHATE. Elza Howdershelt, Moatsville. Guarantee: (As above.) Found: Phos-phoric acid, soluble, II.5I ; reverted, 4.17 ; insoluble, 0.54 ; total, I6.22; available, I5.68.

5OOI. PURE RAW BONE. Elza Howdershelt, Moats- 
ville. Guarantee: Plosphoric acid, insoluble, 22 ; nitrogen, 3.70 ; phosphoric acid from pure animal bone; nitrogen from animal bone. Found: Phosphoric acid, total, 20.80; nitrogen, 3.64; availability of nitrogen, 77 .

Rcmark: Total phosphoric acid low.

50O3. GERMAN POTASH MIXTURE. Elza Howdershelt, Moatsville. Guarantee: Phosphoric acid, available, Io; potash, 2; phosphoric acid from dissolved phosphate rock; potash from German kainit. Found: Phosphoric acid, soluble, 6.95; reverted, 3.43 ; insoluble, o.6o; reverted, ro.98; available, ro.38; potash, 2.15 ; chlorine, 4 .

5005. LIBERTY BELL POTASH MIXTURE. Elza Howdershelt, Moatsville. Guarantec: Phosphoric acid, available, 12; potash, 3; phosphoric acid from dissolved phosphate rock; potash from kainit and manure salts. Found: Phosphoric acid, soluble, 9.27; reverted, 3.88; insoluble, 0.77; total, I3.92 ; available, I3.I 5 ; potash, 3.09 ; chlorine, 2.

\section{IVOOLDRIDGE ORCHILLA COMPANY.}

BALTIMORE, MD.

4954. VIRGINIA BELLE POTASH MIXTURE. W. H. Gwyn, Little Falls. Guarantec: Phosphoric acid, soluble, 9; reverted, 3 ; insoluble, I ; total, I 3 : available, I2: potash, 3 ; phosphoric acid from acid phosphate; potash from manure salt. Found: Phosphoric acid, soluble, 9.79; reverted, 3.53: insoluble. I.47; total. I4.79; available. I3.32; potash, 3.98; chlorine, 2.I.

4955. HIGH GRADE POTATO AND TOMATO GROIVER. IV. H. Gwyn, Little Falls. Guarantee: Phosphoric acid, soluble. 6 ; reverted, 3 ; insoluble, I.50 ; total, ro.5O; available, 9; nitrogen, I.65; potash, 5; phosphoric acid from dissolved bone, tankage and acid phosphate; nitrogen from dissolved bone, tankage and nitrate of soda; potash from muriate of 
potash. Found: Phosphoric acid, soluble, 5.46; reverted, 4.59; insoluble, I.57 ; total, Ir.62 ; available, I0.05 ; nitrogen, r.38 ; potash, 6.04; availability of nitrogen, 79 ; chlorine, I.

Remark: Nitrogen low.

4956. ORCHILLA GUANO. W. H. Gwyn, Little Falls. Guarantee: Phosphoric acid, soluble, 4; insoluble, I3; total, I7; available, 4 ; phosphoric acid from natural bird deposit. Found: Phosphoric acid, soluble, a trace; reverted, 9.49; insoluble, 9.84: total, I9.33; available, 9.49 .

4957. TIGER BONE STOCK PHOSPHATE. W. H. Gwyn, Little Falls. Guarantee: Phosphoric acid, soluble, 5 ; reverted, 3 ; insoluble, I.50; total, 9.50; available, 8 ; nitrogen, I.03; potash, 4.50; phosphoric acid from dissolved bone, tankage and acid phosphate; nitrogen from dissolved bone, tankage and nitrate of soda; potash from muriate of potash. Found: Phosphoric acid, soluble, 5.95; reverted, 3.05 ; insoluble, I.4I ; total, I0.4I ; available, 9 ; nitrogen, I.Io; potash, 5.02 ; availability of nitrogen, 85 ; chlorine, 2.1.

Remark: Chlorine excessive for guarantee.

4958. GERMAN ALKALINE MIXTURE. W. H. Gwyn, Little Falls. Guarantee: Phosphoric acid, soluble, 7 ; reverted, 3 ; insoluble, I.; total, I I ; available, Io; potash, 2 ; phosphoric acid from acid phosphate; potash from manure salt. Found: Phosphoric acid, soluble, 8.66; reverted, 2.65; insoluble, I.II ; total, I2.42; available, I I.3I ; potash, 2.04 ; chlorine, 3.8 .

Remark: Source of potash kainit.

4959. BIZZY BEE. W. H. Gwyn, Little Falls. Guarantee: Phosphoric acid, soluble, 5 ; reverted, 2 ; insoluble, I.5O; total, 8.50 ; available, 7 ; nitrogen, 0.83 ; potash, 1 ; phosphoric acid from dissolved bone, tankage and acid phosphate; nitrogen fron dissolved bone, tankage and nitrate of soda; potash from kainit. Found: Phosphoric acid, soluble, 5.9r ; reverted, 2.44; insoluble, o.49; total, 8.84 ; available, 8.35 ; nitrogen, o.82; potash, I.o3: availability of nitrogen, 78 ; chlorine, 3 .I. 
THE FOLLOWING IS A LIST OF AGENTS WHOSL STOCK WAS SAMPLED DURING I904:

Adams . Keyser

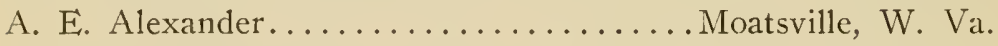
C. A. Anglin . . . . . . . . . . . . . . . . Philippi

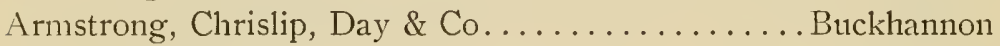
Bishop \& Barbe...................... Jane Lew

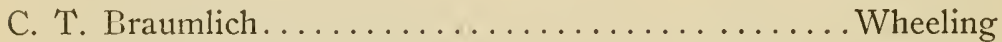

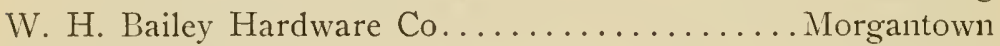

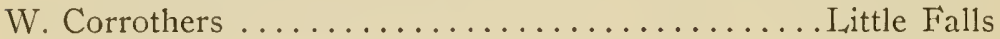

C. R. Carmen....................... Vellsburg

O. R. Carmen........................Wellsburg

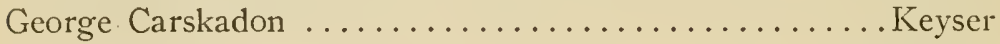

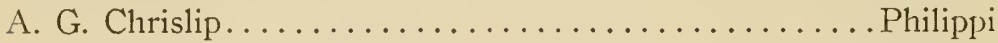

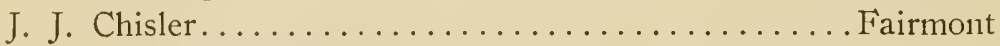

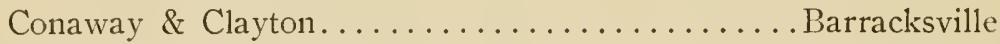

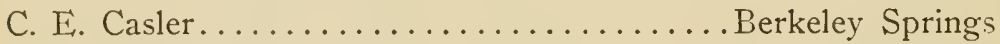
Dean \& Reeves......................... Fairmont

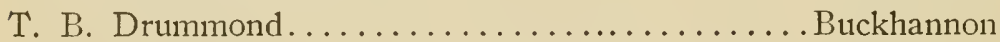
W. R. Dudley....................... Wheeling The Exchange Mill Company................. Grafton

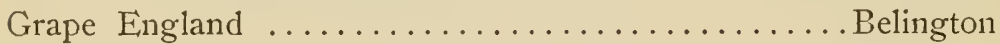

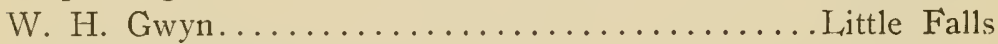

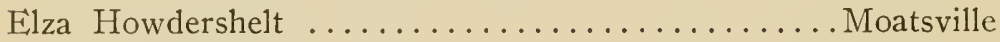

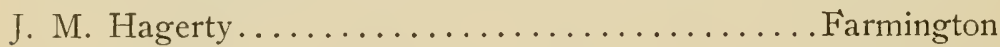

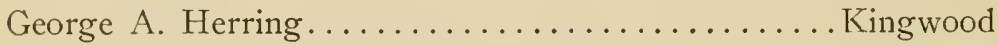
J. L. Hall.......................... Fairmont

R. Hunter. . . . . . . . . . . . . . . Berkeley Springs

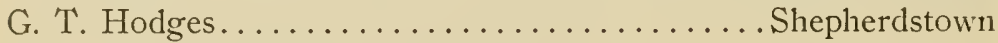
Kane \& Owens Hardware Company..............Weston T. P. Licklider. Martinsburg 


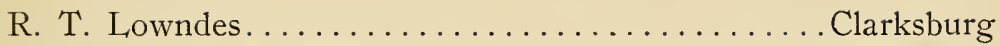

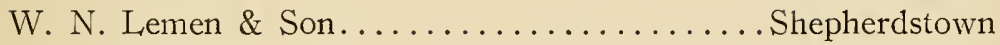

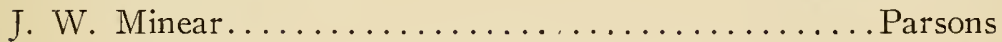

C. W. Mayer \& Son.................... Terra Alta

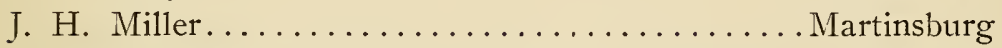

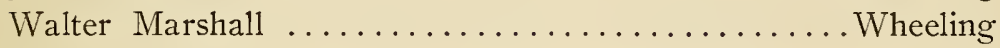

T. A. McNear............................. Lowell

Thomas Nuzum........................Grafton

Cicero Phillips $\ldots \ldots \ldots \ldots \ldots \ldots \ldots \ldots \ldots \ldots$ Belington

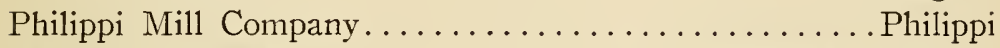

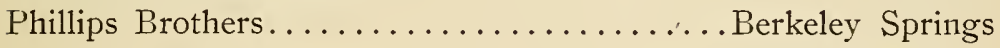

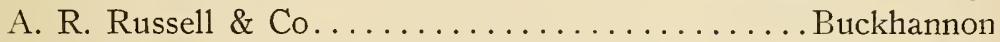

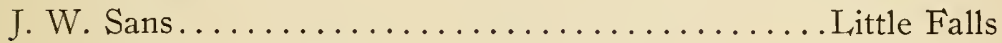

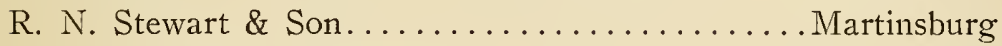

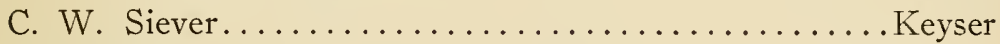

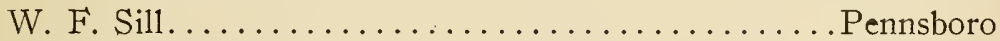

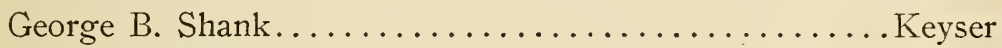

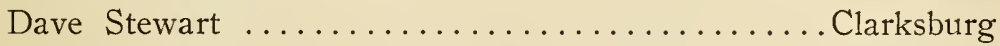

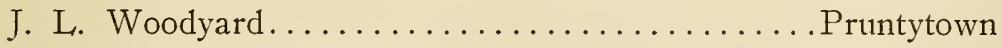

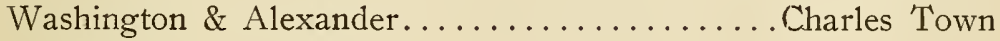


AN ACT CONCERNING COMMERCIAL FERTILIZERS, S'TATE OF WEST VIRGINIA.

AN ACT concerning commercial fertilizers, and repealing chapter seventy-two of the Acts of the Legislature of West Virginia, of eighteen hundred and ninety-one.

\section{[Passed February 2, Igor.]}

Be it enacted by the Legislature of West Virginia :

I. Every person who shall sell, offer or expose for sale, or have in his possession with intent to sell in this state, any com.mercial fertilizer or manure, shall affix conspicuously to every package thereof a plainly printed statement, clearly and truly certifying the number of net pounds of fertilizer in a package, the name, brand or trade-mark under which the fertilizer is sold, the name and address of the manufacturer, the place of manufacture, and stating the percentage of nitrogen or its equivalent in ammonia, or potash, soluble in distilled water and of phosphoric acid in available form, soluble in distilled water, and reverted, as well as the total phosphoric acid, and the materials from which said constituents are derived. In the case of those fertilizers which consist of other cheaper materials, said labels shall give a correct general statement. of the composition and ingredients of the elcments relied upon, contained in the fertilizer which it accompanies.

2. Before any conmercial fertilizer is sold, offered or exposed for sale in this state, the manufacturer, importer or party who catuses it to be sold, exposed or offered for sale, shall file with the Director of the West Virginia Agricultural Experiment Station, under oath, the statement required to be affixed under section one of this act. 
Provided, That when the manufacturer of any brand of fertilizer or manure shall file said sworn statement no agent or dealer shall be required to file such statement. The making of any affidavit required by this chapter falsely shall be perjury.

3. The manufacturer, importer, agent or seller of any brand of commercial fertilizer or material used for manurial purposes shall pay for each brand at the time he files a sworn statement required by section two of this act a registration fee of one dollar per annum.

4. The registration fee required to be paid by section three of this act shall be paid to the treasurer of the West Virginia University for the use of the Agricultural Experiment Station, and the party making such payment shall take from the said treasurer triplicate receipts therefor, one of which he shall retain, and the others shall be deposited, one with the Director of the Agricultural Experiment Station, and the other with the secretary of the Board of Regents, and by them filed and preserved in their respective offices.

Immediately after the filing of the receipts aforesaid, with the Director of the Agricultural Experiment Station, said Director shall issue a certificate to the party making such payment, stating the amount of fees paid, and the name, brand or trade-mark under which the fertilizer is sold, the name and address of the manufacturer or importer, the place of manufacture, and that the applicant for said certificate is authorized to sell said fertilizer within the State of West Virginia for the period of one year from the first day of January to the thirty-first day of December, inclusive. Said certificates may be issued during the month of December for the year commencing on the first day of January thereafter.

6. It shall be the duty of the Director of the West Virginia Agricultural Experiment Station to print or cause to be printed a label or tag setting forth the sworn statement required in section two of this act. The Director shall furnish such labels or tags in quantities of one hundred or multiples thereof, to any person complying with the requirements of this act, and desiring to sell, offer or expose for sale any commercial fertilizer in this State, and 
shall receive pay therefor at the rate of forty cents for such number of labels or tags as may be required to be affixed to one ton of fertilizer, and shall without delay pay the same to the treasurer of the West Virginia University, for use of the Agricultural Experiment Station, and take duplicate receipts therefor, one of which he shall retain, and the other he shall deliver to the secretary of the Board of Regents, who shall file and preserve the same in his office.

7. The Board of Regents of the West Virginia University shall expend the money received under the provisions of this act in meeting the legitimate expenses of the station in making analyses of fertilizers, in experimental tests of same, and in such other experimental work and purchases as shall inure to the benefit of the farmers of this State, and shall include in their annual report a statement of the receipts and disbursements thereof.

8. It shall be the duty of the Director of the said Experiment Station, in person or by deputy, to take samples for analysis from any lot or packages of any fertilizer or manure which may be in the possession of any dealer in the State, and he is hereby authorized to prescribe and enforce such rules and regulations as he may deem necessary to carry fully into effect the true intent and meaning of this act; and any agriculturalist, purchaser of any fertilizer or manure in this State may take a sample of the same under the rules and regulations of the Director of the said Experiment Station and forward the same to him for analysis, and if the Director has reason to believe that the manufacturer of, or dealer in, such fertilizer or manure has made any false or fraudulent representations in regard to said fertilizer or manure, he shall cause the said sample to be analyzed free of charge, and certify the same to the person forwarding the sample. And it shall be the duty of every agent or dealer in fertilizers or manures, within the meaning of this act, in this State, when ordering consignments thereof, to promptly notify the Director of the said Experiment Station of the amount ordered, and the place and approximate time of delivery of said fertilizers and manures.

9. Said Director shall also publish by bulletin the brand, name and location of the manufacturer and chemical analysis of every 
fertilizer or manure analyzed or caused to be analyzed by him. Said last publication to be made, if practicable, before the time at which said fertilizer is to be applied to the soil.

IO. Any manufacturer or vendor of any fertilizer or manure who shall sell or offer or expose for sale any fertilizer or manure without having previously complied with the provisions of this act as hereinbefore set forth, shall be deemed guilty of a misdemeanor, and upon conviction thereof shall be fined not less than fifty nor more than five hundred dollars.

I I. Any company, firm or corporation who shall wilfully remove from or deface or change any label, or tag, or brand affixed to any package of fertilizer under the provisions of this act before such fertilizer has been used for manurial purposes, or who shall sell such fertilizer without such label or tag being affixed thereto at the time of the sale, shall be deemed guilty of a misdemeanor, and upon conviction thereof shall be fined not less than ten nor more than fifty dollars for each offense.

I2. Any company, firm or person who shall remove from or cause to be removed from any package of commercial fertilizer or manure any statement. label or tag affixed thereto under the provisions of this act, and affix or cause the same to be affixed to any other package of commercial fertilizer or manure, shall be deemed guilty of a misdemeanor, and upon conviction thereof shall be fined not less than ten nor more than fifty dollars for each offense.

I3. Any company, firm or person violating any of the provisions of this act, or who fails to comply with any of the requirements of this act, shall be deemed guilty of a misdemeanor. and upon conviction thereof shall, when no other penalty is prescribed, be fined not less than ten nor more than one hundred dollars for each offense. But this act shall not be construed to apply to any one who manufactures fertilizers for his own use and not for sale.

I4. The Director of said Experiment Station shall report to the prosecuting attorney of the county where the offense was committed all violations of the provisions of this act, and failure to comply therewith, and a copy of any label, statement or tag re- 
quired to be filed with said Director or prepared by him, and any analysis made or caused to be made by him, when duly certified by said Director, shall be received in evidence in any prosecution or suit for any violation of the provisions of this act.

I, Harry Shaw, Clerk of the House of Delegates of West Virginia, hereby certify that the paper hereto annexed is a true copy of an Act of the Legislature of West Virginia, passed on the second day of February, I90I; and that the same takes effect ninety days from the date of its passage.

Given under my hand this March 20th, I9OI.

HARry Shaw, Clerk of the House of Delegates. 

